\title{
Single-Spin Magnetic Resonance in the Nitrogen-Vacancy Center of Diamond
}

\author{
Dieter Suter \\ Fakultät Physik, TU Dortmund, \\ 44221 Dortmund, \\ Germany \\ Fedor Jelezko \\ Institut für Quantenoptik, \\ Universität Ulm, Ulm, \\ Germany
}

(Dated: December 12, 2016)

\begin{abstract}
Magnetic resonance of single spins has flourished mostly because of the unique properties of the NV center in diamond. This review covers the basic physics of this defect center, introduces the techniques for working with single spins and gives an overview of some applications like quantum information and sensing.
\end{abstract}

\section{CONTENTS}

I. Introduction

II. The Nitrogen-Vacancy (NV) Center

A. Defects in diamond

B. Electronic structure

C. Ion implantation

D. Confocal microscopy

III. Optically Detected Magnetic Resonance

A. Optically detected cw-ESR

B. Time-resolved ESR

1. Initialization

2. Optical detection

3. Rabi oscillations

4. Free induction decay

C. Interactions of the electron spin

1. Hyperfine couplings

2. Relaxation

IV. Nuclear Magnetic Resonance

A. Experimental Procedure

B. Nuclear Spin Interactions

1. ${ }^{14} \mathrm{~N}$ Hyperfine Interaction

2. ${ }^{14} \mathrm{~N}$ Quadrupole Interaction

3. ${ }^{14} \mathrm{~N}$ nuclear Zeeman interaction

4. ${ }^{13} \mathrm{C}$ Hyperfine

C. Enhanced Nuclear Magnetism

V. Quantum Information

VI. Sensing

VII. Conclusions and Outlook

Acknowledgments

References

\section{INTRODUCTION}

Nuclear magnetic resonance (NMR) is usually characterized as a spectroscopic technique that provides very detailed information on almost every possible substance but also as very insensitive: typical NMR experiments use samples with some $10^{20}$ spins. This may be contrasted to atomic physics experiments, where optical detection of single atoms or ions was demonstrated as early as 1980 (Neuhauser et al., 1980), less than 30 years after Schrödinger wrote "In the first place it is fair to state that we are not experimenting with single particles, any more than we can raise Ichthyosauria in the zoo" (Schrödinger, 1952). The excellent sensitivity of optical spectroscopy was also demonstrated by the detection of single molecules in condensed matter (Moerner and Kador, 1989; Orrit and Bernard, 1990). An extension of these techniques also made it possible to detect magnetic resonance transitions in the same molecules first of the electron spins (Köhler et al., 1993; Wrachtrup et al., 1993) and subsequently also of single nuclear spins (Wrachtrup et al., 1997).

While most of these pioneering experiments were done with aromatic molecules at low temperatures, similar techniques were also applied to the study of defect centers in inorganic materials (Spaeth et al., 1992). The two directions met in 1997, when the first report of single spin magnetic resonance at room temperature was published (Gruber et al., 1997), using the nitrogen-vacancy (NV)center in diamond. This breakthrough work attracted lots of attention as it opened a new path that made work with single spins comparatively easy and accessible to many groups without having to use low-temperature setups. Apart from the fundamental interest in NV centers, it also opened a number of interesting applications, such as quantum information processing and communication or sensing with nanometer-resolution.

Diamond is known as a unique material for applications where hardness, high thermal conductivity or charge carrier mobility play a crucial role. Natural diamond resources are limited and natural crystals are created in very different environments leading to large vari- 
ation of their physical properties. This makes technological applications of natural material difficult. Five decades ago material scientists were able to show that diamond crystals can be created artificially. First it was done using high pressure and high temperature (HPHT) by bringing carbon-containing starting materials to a point where diamond is the stable phase (Bundy et al., 1955). More recently diamond was synthesized using chemical vapor deposition (CVD) from plasmas containing a mixture of hydrogen and methane (Matsumoto et al., 1982). The CVD process relies on growth under conditions where the diamond phase is metastable; growth becomes possible if the etching rate of the graphitic phase by the hydrogen plasma exceeds the etching rate of the diamond phase. Under such conditions, the kinetics of the growth reaction allows diamond to be grown also at temperatures and pressures where graphite is the more stable form of carbon. The ability to create artificial diamond and control its composition including isotopic modifications allowed one to understand the formation and structure of many diamond defects and opened the way to applications of diamond in different technologies.

This review is structured as follows: chapter II describes the basic properties of the NV center and the experimental techniques that were developed to work with single centers. Any experiment on single NV centers has to start with the electron spin. Accordingly, chapter III focuses on the electron spin and the techniques used to drive and detect its transitions. Chapter IV continues to nuclear magnetic resonance of the ${ }^{13} \mathrm{C}$ and ${ }^{14} \mathrm{~N}$ nuclear spins that are in contact with the electron spin of the NV center. Chapters V and VI focus on applications to quantum information and sensing, and the review concludes with chapter VII.

\section{THE NITROGEN-VACANCY (NV) CENTER}

\section{A. Defects in diamond}

Studies of different types of diamond have revealed several hundred defects (Zaitsev, 2001, 2000). Many of them exhibit strong optical transitions between electronic states located within the band gap of diamond. Such defects are often called color centers. They play important roles in the growing field of quantum technologies. Each color center creates an absorption band and can result in strong coloration of diamond crystals at high defect concentrations. The most common defect present in natural diamond is a single substitutional nitrogen, the socalled P1 center. Its concentration in artificial crystals can reach several hundred parts per million (ppm). When present in such high concentration, this defect causes a yellow coloration of the diamond. The absorption of the $\mathrm{P} 1$ center is related to its ionization, i.e. the transition between its localized ground state and the delocalized states of the conduction band.

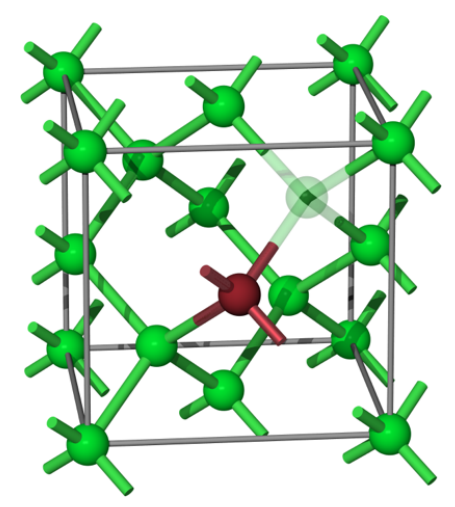

FIG. 1 Structure of the NV center.

The Nitrogen-Vacancy (NV) center, which is the focus of this article, is a defect in which one of the carbon atoms is substituted by a nitrogen atom, while one of its four neighbors is missing, i.e. the site is vacant. The lone pair of the nitrogen is oriented towards that vacancy and overlaps with the three dangling bonds of the carbon atoms located next to the vacancy. The defect conserves the $C_{3}$ symmetry axis of the diamond lattice that joins the $\mathrm{N}$ and $\mathrm{V}$ sites, along the [111] direction.

\section{B. Electronic structure}

The defect exists in at least two charge states: negative and neutral. If the defect is neutral, the ground state of the five electrons (2 from the N, 3 from the dangling carbon bonds) has a spin $S=1 / 2$. In this state, the NV center does not lend itself to optically detected magnetic resonance, since the dynamic Jahn-Teller effect causes rapid relaxation of the ground state spin sublevels (Felton et al., 2008). Here, we are concerned primarily with the negatively charged $\mathrm{NV}^{-}$center, where the NV has captured an additional electron. The ground state has then a spin $S=1$ and a strong optical transition between the ${ }^{3} A$ ground state and the ${ }^{3} E$ excited states. The emission occurs in the visible part of the spectrum, with a zero-phonon line at $637 \mathrm{~nm}(1.945 \mathrm{eV})$ and a phonon sideband extending to $800 \mathrm{~nm}$. Allowed optical transition and high fluorescence quantum yield are crucial for single site detection using confocal microscopy techniques (Jelezko and Wrachtrup, 2004). 96\% of the fluorescence photons are emitted into the phonon sidebands, only a few percent in the zero-phonon line. The width of the zero-phonon line is limited by the decay process of the excited state (11.5 ns) for high quality diamond, which is characterized by low strain and low impurity concentration (Batalov et al., 2008). At higher defect concentrations, the line becomes broader. 
Although the NV center has been studied for more than five decades and many of its properties have been established, there are still open questions related to its energy level structure. In particular the absolute energy of the singlet levels has not been determined, but their separation is known. There are also uncertainties concerning the stability of its charge state, especially for NV centers located close to the surface. Under visible illumination, the NV center exists in a dynamic equilibrium between the neutral and negative charge states. The negatively charged NV center can be ionized by two-photon excitation leading to its transformation into the neutral state. When excited by light with a wavelength shorter than $575 \mathrm{~nm}$ (corresponding to the zero-phonon line of the neutral state), the optical excitation leads to a recovery of the negative charge state via a two photon process involving capturing of an electron from the valence band (Siyushev et al., 2013). The equilibrium between these two charge states can be controlled by the laser excitation wavelength or by the doping of diamond. Continuous excitation of negatively charged NV center with wavelengths longer than $575 \mathrm{~nm}$ shifts the equilibrium towards the neutral state. The negative state can be stabilized using doping of diamond by phosphorus, which is a shallow donor with an activation energy of $0.5 \mathrm{eV}$ (Doi et al., 2016).

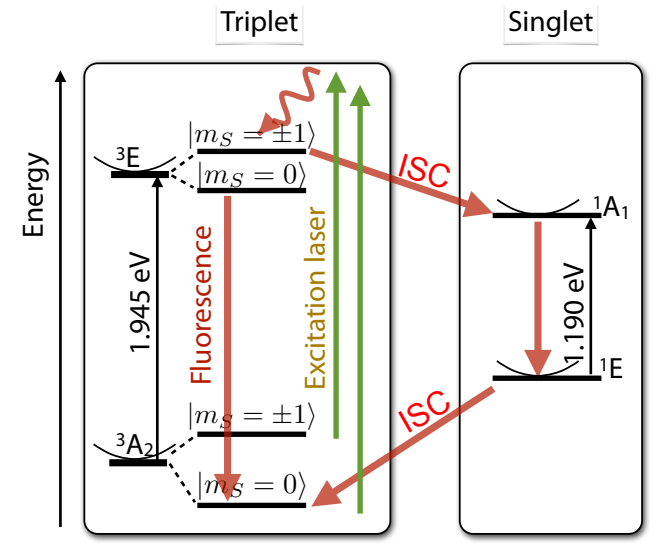

FIG. 2 The most important electronic states of the $\mathrm{NV}^{-}$ center. The numbers refer to the transition energies of the zero-phonon lines, the parabola indicate the continuum of vibrationally excited states. $m_{S}$ refers to the electron spin. ISC = intersystem crossing.

Figure 2 shows the relative energies of the most important electronic states of the $\mathrm{NV}^{-}$center. The ${ }^{3} A_{2}$ ground state is connected to the excited triplet state ${ }^{3} E$ via an allowed optical transition with a photon energy of 1.945 $\mathrm{eV}$. In most experiments, the system is excited by a green laser. The photon energy of $2.33 \mathrm{eV}$ drives the system well above the ${ }^{3} E$ state. From there it quickly relaxes nonradiatively down to the ${ }^{3} E$ state, typically without changing its spin state. The radiative relaxation down to the ground state depends on the spin: while the $m_{S}=0$ state most often $(\approx 89 \%)$ relaxes directly to the ground state, the $m_{S}= \pm 1$ states often $(\approx 45 \%)$ (Dumeige et al., 2013) cross over into the singlet manifold, which consists of two states whose energies are between those of the two triplet states (see fig. 2). From there they eventually also relax back to the triplet ground state. However, during the intersystem-crossing (ISC) between the triplet to the singlet manifolds, the electronic spin is not conserved and with a probability of $\approx 45 \%$, the system ends up in the $m_{S}=0$ state. Since the whole process of crossing through the singlet manifold requires more time than direct relaxation (Acosta et al., 2010; Dumeige et al., 2013), this reduces the number of photoluminescence (PL)-photons emitted per unit time. The fluorescence count is therefore a possible method for determining the spin state of the $\mathrm{NV}^{-}$center (Nizovtsev et al., 2001). After several excitation cycles, the system always ends up in the $m_{S}=0$ state (Harrison et al., 2004; van Oort and Glasbeek, 1991). Accordingly this procedure drives the system into the $m_{S}=0$ ground state. If used as a readout process, this procedure is destructive, since the memory of its previous state is lost.

At low temperature with selective excitation into the zero-phonon line, the situation changes. Under these conditions, spin selective excitation results in different mechanisms and contrast of the optically detected magnetic resonance (ODMR) signal. The contrast becomes positive, since the applied microwave field brings the NV centers back to resonance with the exciting laser (van Oort et al., 1988). Long cycling transitions of the NV center allow single shot readout of ODMR signal of single NV centers (Robledo et al., 2011). Spin selective excitation at cryogenic temperatures also allows coherent population trapping (Santori et al., 2006) enabling robust purely optical control of the spin state (Yale et al., 2013).

\section{Ion implantation}

NV centers are present in naturally occurring diamonds as well as in industrially manufactured samples. For synthetic crystals grown by chemical vapor deposition the concentration of NV centers is typically about $1 \%$ of the concentration of $\mathrm{P} 1$ centers. It is possible to convert P1 centers into NV centers by electron or ion irradiation and annealing (Martin et al., 1999).

NV centers can also be generated in ultra-pure diamond by implantation of nitrogen ions and subsequent annealing. The ions create substitutional nitrogen atoms as well as vacancies. At temperatures above $700{ }^{\circ} \mathrm{C}$, the vacancies become mobile. Some of them are trapped by nitrogen, others annihilate with interstitials or disappear when reaching the diamond surface. The conversion yield of nitrogen to NV centers depends critically on the number of vacancies created during the implantation and 
therefore on the implantation energy. It can reach several tens of percent for $\mathrm{MeV}$ implantation but is only a few percent for keV implantation (Pezzagna et al., 2011).

The technique of nitrogen implantation is crucial for positioning single defects for quantum information and quantum sensing applications. The defects can be created one by one by implanting individual ions (Meijer et al., 2005; Rabeau et al., 2006), and this approach can be scaled up using nanoscale masks made by electron beam lithography (Toyli et al., 2010). The depth can be controlled by the implantation energy and molecular dynamics simulations can provide a good estimate of average distance of implanted NV centers from the diamond surface (Antonov et al., 2014; Lehtinen et al., 2016). Ion implantation with conventional ion sources are subject to statistical fluctuations of the number of ions implanted per spot, which becomes particularly strong for small numbers. Accordingly, there is a significant effort for deterministic ion implantation, which would allow precise control of the number of implanted ions (Schnitzler et al., 2009). If lateral positioning of the defects is not required, NV centers can also be created in a thin layer by briefly adding nitrogen to the growth chamber during during CVD growth (Ohashi et al., 2013; Ohno et al., 2012; Fávaro de Oliveira et al., 2016), in a technique known as delta-doping.

The depth of implanted ultra-shallow (less than 10 $\mathrm{nm}$ depth) NV centers can be characterized by coherence associated with nuclear spins on the diamond surface (Pham et al., 2016). Optical techniques including superresolution microscopy (Pezzagna et al., 2010; Rittweger et al., 2009) and localization microscopy (Häußler et al., 2014) also allow one to characterize the location of NV centers with nanometer precision allowing a large dynamic range.

\section{Confocal microscopy}

Initialization of the spin state is achieved by irradiation with suitable light, typically from a laser. Detection of the spin polarisation requires efficient collection of the emitted photons from the region of interest. For both tasks, a microscope objective is helpful, as it focuses the laser on the region of interest and collects the photons from this region. If high spatial resolution is required, specifically if the region of interest consists of a single center, confocal microscopy is an effective tool for excitation and detection.

Figure 3 shows the basic setup for confocal microscopy. The incoming green laser beam is reflected by a dichroic mirror and focused onto the sample by a microscope objective lens with high numerical aperture. The emitted photons, which have a longer wavelength than the incoming laser light, are collected by the same lens. The dichroic mirror is designed to transmit most of the photo-

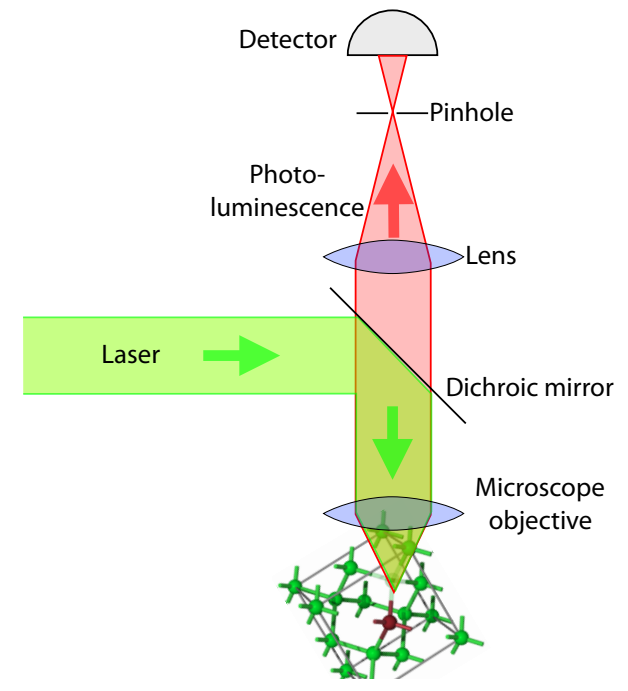

FIG. 3 Setup for confocal microscopy.

luminescence (PL), which is then focused with a second lens through a pinhole onto the detector. The pinhole suppresses light that was emitted in a different part of the sample and therefore not focused onto the pinhole. Compared to a conventional microscope, the confocal microscope has a much higher depth-resolution. The detector is usually an avalanche-photodiode (APD), optimized for single photon counting. Typical quantum efficiencies are of the order of $75 \%$.

\section{OPTICALLY DETECTED MAGNETIC RESONANCE}

\section{A. Optically detected cw-ESR}

The Hamiltonian of the electron spin $\vec{S}(S=1)$ in the $\mathrm{NV}$ center can be written as

$$
\mathcal{H}_{S}=D S_{z}^{2}-\gamma_{e} \vec{B}_{0} \cdot \vec{S}
$$

with the zero-field splitting $D=2.870 \mathrm{GHz}, \gamma_{e}=-28.02$ $\mathrm{GHz} / \mathrm{T}, \vec{B}_{0}$ is the static magnetic field and the $z$-axis is chosen along the symmetry axis of the center. In zero magnetic field, the center therefore has 2 degenerate transitions at $2.870 \mathrm{GHz}$, from the $m_{S}=0$ ground state to the two $m_{S}= \pm 1$ states. As shown in Fig. 4, the two transitions become non-degenerate in a finite magnetic field.

Since the rate at which the NV center emits photons depends on its spin state, it is possible to use the photoluminescence as a sensitive detector of magnetic resonance. If the center is continuously irradiated with a green laser, it initializes the spin into the $m_{S}=0$ state. In this state, the center emits the highest number of photons per unit time. If a resonant microwave field is applied to the system, it causes transitions from the $m_{S}=0$ to the $m_{S}= \pm 1$ states, thereby reducing the count rate. 


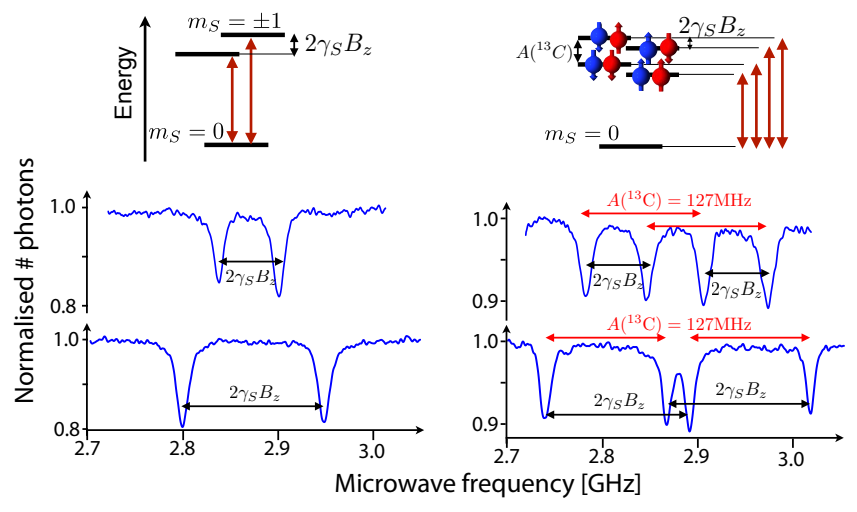

FIG. 4 Optically detected ESR spectra of two different NV centers. The spectra shown in the left-hand part show a pair of lines separated by the Zeeman interaction, while the spectra on the right-hand part show additional splittings due to the hyperfine interaction between the electron spin and a ${ }^{13} \mathrm{C}$ nuclear spin in the first coordination shell.

Fig. 4 shows four ODMR spectra from two different NV centers, measured in two different fields. The center shown on the left-hand part has no resolved couplings to nuclear spins, while the center on the right-hand side includes a ${ }^{13} \mathrm{C}$ nuclear spin next to the vacancy. The hyperfine interaction between this nucleus and the electron spin splits the resonance by $127 \mathrm{MHz}$. The upper row was measured in a magnetic field of $B_{0} \approx 1.1 \mathrm{mT}$, while the lower ones used a field of $2.7 \mathrm{mT}$.

This optical detection scheme can also be applied to the detection of nuclear spin transitions. Using a detection scheme similar to a quantum-non-demolition measurement (Grangier et al., 1998), based on selective microwave irradiation and photoluminescence photon counting, it is possible to detect the state of a single nuclear spin with high probability in a measurement time of $\approx 5$ ms (Neumann et al., 2010a).

ODMR can also be used to probe the electronically excited state - in this case the ${ }^{3} E$ state. At room temperature fast phonon-induced relaxation processes lead to the appearance of a single broad ODMR line associated with the excited state (Neumann et al., 2009). The width of this line is related to the fast radiative decay of the excited state. ODMR transitions associated with the ground state spins are free from this type of broadening.

\section{B. Time-resolved ESR}

\section{Initialization}

The initial condition for time-resolved ESR experiments is usually prepared by a green laser pulse, with a duration of 1-5 $\mu \mathrm{s}$. This results in the predominant population of the $m_{S}=0$ ground state. There is some debate about the polarisation that is reached in this pro- cedure, with values reported over the range of $42 \%-96 \%$ (Doherty et al., 2013).

While the optical cycling through the excited state predominantly affects the electron spin, it also modulates the hyperfine interaction and therefore enhances the relaxation of the nuclear spin. If the laser pulse duration exceeds a few $\mu \mathrm{s}$, the resulting state leaves the nuclear spin completely unpolarized.

\section{Optical detection}

The optical detection scheme discussed in section III.A can be applied to time-resolved experiments. In this case, detection is performed by applying a laser pulse at the end of the experiment. The measured photon count rate can then be translated into a measurement of the spin populations.

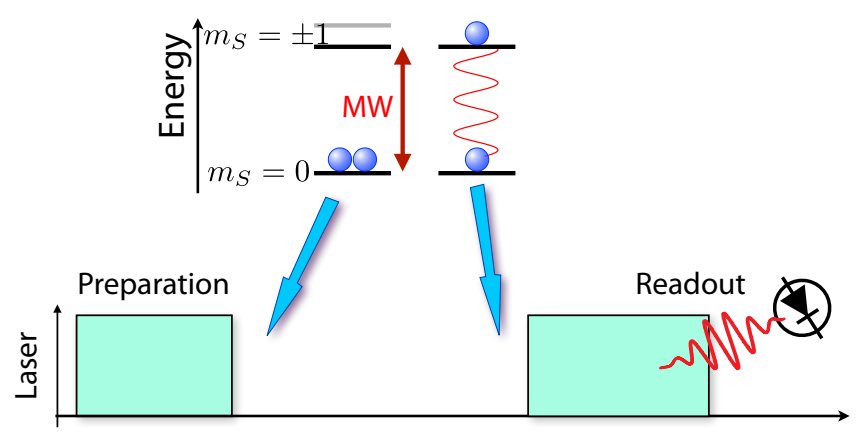

FIG. 5 Basic pulse sequence for time-resolved experiments; $\mathrm{MW}=$ microwave.

Figure 5 illustrates the principle: A first laser pulse initializes the spins into the $m_{S}=0$ ground state. After the time-resolved experiment, typically driven by a sequence of microwave pulses, the readout pulse measures the remaining population of the $m_{S}=0$ state.

Since the laser also initializes the spin into the $m_{S}=0$ state not only during preparation, but also during readout, this measurement is destructive, i.e. it changes the state. As a result, it is not useful to count photons indefinitely, and the best results are obtained for a measurement time that is comparable to the time required to pump the system into the ground state. The optimal duration of the readout process is therefore determined by the rates at which the two states scatter photons and the rate at which the spin is pumped from the $m_{S}= \pm 1$ to the $m_{S}=0$ state.

If the system is initially in state $|0\rangle$, it scatters photons at a rate $r_{0}$. If it is initially in state $| \pm 1\rangle$, the scattering rate is $r_{1}<r_{0}$. While scattering photons, it has a probability of changing to state $|0\rangle$, which can be quantified by a rate $k$. Accordingly, if the system is initially in state $| \pm 1\rangle$, the probability that it remains in this state decays as $p_{1}=e^{-k t}$ and the population of state $|0\rangle$ increases as 
$p_{0}=1-e^{-k t}$. Accordingly, the count rate increases as

$$
r(t)=r_{1} e^{-k t}+r_{0}\left(1-e^{-k t}\right) .
$$

If we count photons for pulses of increasing duration $\tau$, we expect to obtain

$$
\begin{aligned}
& N_{0}(\tau)=r_{0} \tau \\
& N_{1}(\tau)=\int_{0}^{\tau} r(t) d t=r_{0} \tau-\left(r_{0}-r_{1}\right) \frac{1-e^{-k \tau}}{k}
\end{aligned}
$$

photons if the system is initially in state $|0\rangle$ or $| \pm 1\rangle$.

The rate $r_{0}$ can be determined by fitting $N_{0}$ vs. $\tau$. Similarly, $k$ and $r_{1}$ can be found by fitting

$$
N_{0}(\tau)-N_{1}(\tau)=\frac{r_{0}-r_{1}}{k}\left(1-e^{-k \tau}\right) .
$$

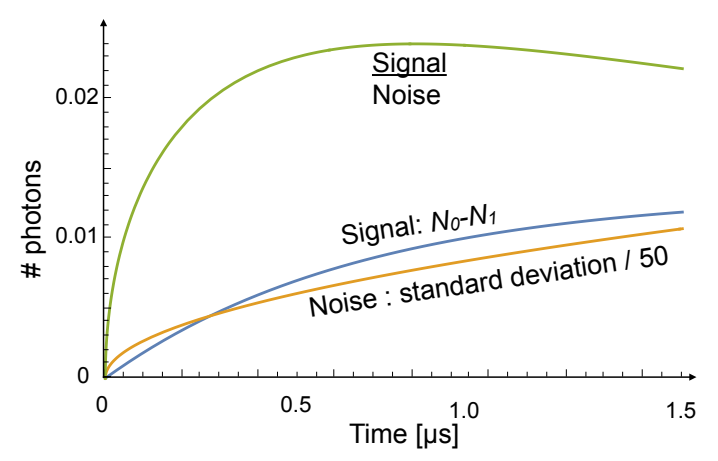

FIG. 6 Number of detected photons during a single laser pulse as a function of the counting period. The noise is due to shot noise (Poissonian statistics). It has been divided by a factor of 50 to fit onto the same scale. The signal-to-noise ratio peaks for a counting period of approx. $0.8 \mu \mathrm{s}$.

As shown in figure 6 , the difference signal $N_{0}(\tau)-$ $N_{1}(\tau)$ increases monotonically with the duration $\tau$ of the counting period, but saturates for times $\tau \gg k^{-1}$. The shot noise, however, which is proportional to the total number $N_{0}(\tau)+N_{1}(\tau)$ of photons, increases without limit. Accordingly, the signal-to-noise ratio goes through a maximum for counting periods $\approx k^{-1}$. Typical numbers are $r_{0}=1.0 \cdot 10^{5} / \mathrm{s}, r_{1}=0.80 \cdot 10^{5} / \mathrm{s}$, and $k=1.5 \mu \mathrm{s}^{-1}=1 / 667 \mathrm{~ns}$. With these numbers, the highest contrast to noise ratio is obtained for a pulse duration of 838 ns. For shorter pulses, the number of detected photons is too small, for longer pulses, the spin has been reset into the $m_{S}=0$ state and the additional counts do not contain any information. In practice, it is advantageous to count photons also during a later period, when the center is in the ground state. The ratio of the two count values is less sensitive to fluctuations of the laser intensity or drifts of the position of the microscope. The detection efficiency can be improved under specific conditions, e.g. by working at a magnetic field (strength and orientation) where the excited state undergoes a level anti-crossing (Steiner et al., 2010).
3. Rabi oscillations
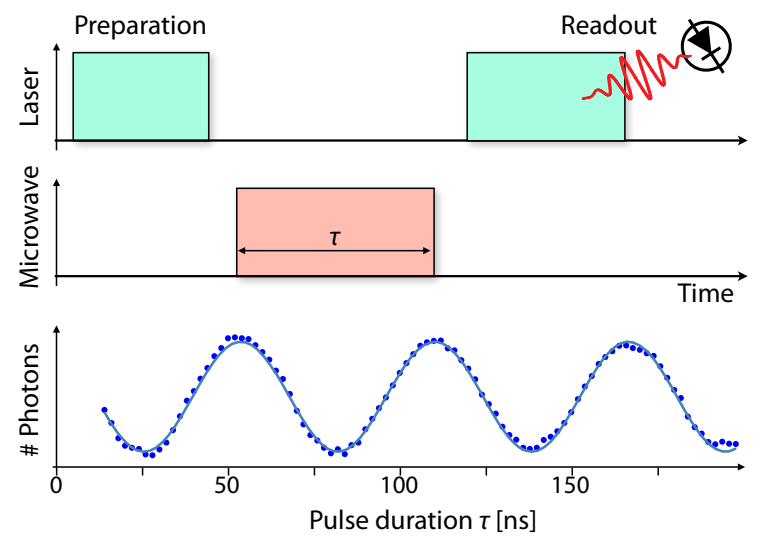

FIG. 7 Pulse sequence for measuring Rabi oscillations and experimental observation.

The simplest time-resolved experiment consists of a single microwave pulse, sandwiched between two laser pulses (see Fig. 7). The first laser pulse initializes the system into the $m_{S}=0$ state, where the count rate reaches a maximum. The microwave pulse then drives the $m_{S}=0 \leftrightarrow m_{S}=1$ transition, thus periodically reducing the count rate. The minimum of the count rate corresponds to the situation when the spin is in the $m_{S}=1$ state. During the second laser pulse, the photons are counted and the result is plotted against the pulse duration $\tau$, as shown in the bottom trace.

An interesting aspect of single-spin magnetic resonance is that the Rabi oscillations are not subject to inhomogeneous decay. The observed decay times are typically also longer than $T_{2}$, since the applied microwave field decouples the spin from the nuclear spin noise that is the main source of coherence decay in FID experiments. Accordingly, Rabi oscillations can persist for very long times close to the limit given by the longitudinal relaxation time $T_{1}$.

\section{Free induction decay}

The basis for time-resolved ESR is the measurement of a free induction decay.

Figure 8 shows the basic pulse sequence used for measuring free-precession signals. The first laser pulse initializes the spin into the $m_{S}=0$ state. The microwave pulse generates a superposition state, e.g. $(|0\rangle+|1\rangle) / \sqrt{2}$. During the free precession period, this state acquires a phase $\phi(\tau)=\left(\mathcal{E}_{1}-\mathcal{E}_{0}\right) \tau / \hbar$, where $\mathcal{E}_{i}$ are the energies of the states. The second microwave pulse converts this state back into populations of the $m_{S}=0$ and $m_{S}=1$ states. The populations are modulated by $\cos \phi(\tau)$. During the second laser pulse, the photon count measures this population, and the resulting signal is shown in the bottom 

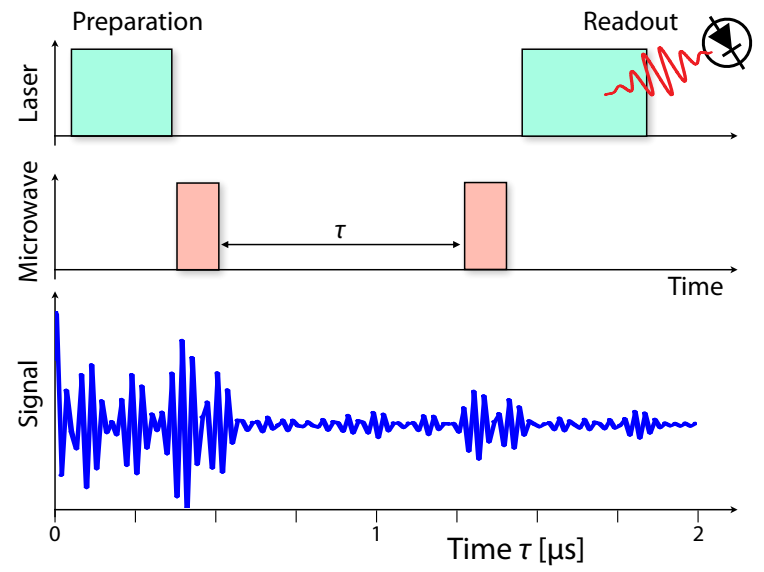

FIG. 8 Free induction decay of a single NV center measured by the Ramsey-fringe method.

trace, which is recorded by incrementing the delay $\tau$.

Compared to conventional magnetic resonance, where FID's are usually measured by an inductive detector, this type of detection is significantly more time-consuming, since every point of the FID has to be measured indirectly, by converting the coherence into a population that must be read out. This type of detection is often termed a Ramsey scheme, after Ramsey's experiment with separated radio frequency fields (Ramsey, 1950).

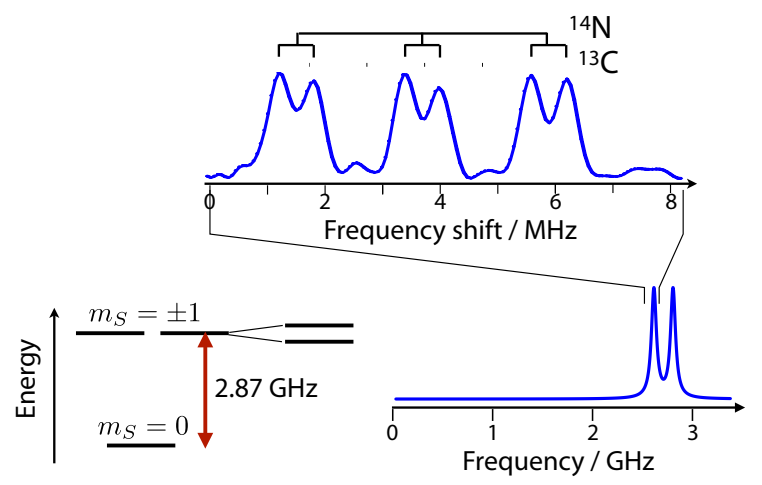

FIG. 9 Example of an ESR spectrum obtained by Fouriertransformation of the FID shown in figure 8.

Like in conventional magnetic resonance, the spectrum is obtained as the Fourier transform of the free induction decay signal. In the bottom part, Figure 9 shows a schematic overview of the spectrum, which consists of two resonance lines around $2.9 \mathrm{GHz}$. These lines correspond to the transitions from the $m_{S}=0$ ground state to the $m_{S}= \pm 1$ states. The center of the doublet is given by the zero-field splitting of $2871 \mathrm{MHz}$, while the splitting corresponds to twice the Zeeman interaction. The top trace, which is the Fourier transform of the free precession signal shown in figure 8 , shows the spectral region of the low-frequency transition. The single line of the ESR spectrum is split by the hyperfine interaction between the electron spin and two neighboring nuclear spins. The ${ }^{14} \mathrm{~N}$ nuclear spin $(I=1)$ of the NV center splits it into 3 lines, and the hyperfine interaction with a ${ }^{13} \mathrm{C}$ nuclear spin $(I=1 / 2)$ generates an additional splitting into a total of 6 lines .

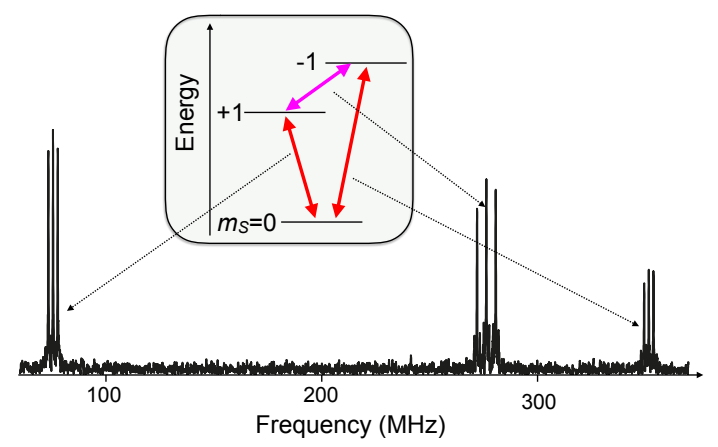

FIG. 10 Broadband ESR spectrum measured with pulsed excitation, together with the relevant energy level scheme. The reference frequency was $2671 \mathrm{MHz}$.

If the pulsed excitation has a sufficiently large bandwidth, it can excite not only the two transitions from $m_{S}=0$ to $m_{S}= \pm 1$, but also the double quantum transition between the $m_{S}= \pm 1$ states(Niemeyer et al., 2013). Since the dominant interaction is the zero-field splitting, the resonance frequency of this double quantum transition is, in weak to moderate magnetic fields, smaller than that of the single-quantum transitions.

In a Ramsey-type FID measurement, the observed oscillation frequency of the signal is given by the difference between the transition frequency of the coherence and the frequency of the microwave source that generates the pulses. If this frequency is in the middle of the spectrum, the resulting spectrum is folded around the microwave frequency, which can be inconvenient. This can be avoided either by performing two measurements, with the phase of the readout pulse shifted by 90 degrees (quadrature detection), or by switching the reference frequency of the microwave to a value that is more suitable for detection, e.g. below the lowest frequency. This is the path most often used. In the spectrum shown in figure 10, this reference frequency was $2671 \mathrm{MHz}$, just off the shown trace. The single-quantum transitions appear at 75 (=2746-2671) MHz and 352 (=3023-1671) MHz, while the double quantum transition appears at the difference between them, which is close to 3023-2746 $=352-75=$ $277 \mathrm{MHz}$.

While most experiments on NV centers are performed in weak magnetic fields of a few $\mathrm{mT}$ or less, there is also some work in the high-field range above 1 Tesla. This is useful, e.g., when NV's are used for sensing nuclear spins and the field should be strong enough, e.g., to distinguish between spin species through their chemical shifts (Stepanov et al., 2015). 


\section{Interactions of the electron spin}

1. Hyperfine couplings

As shown in figure 9, (almost) every center shows a hyperfine splitting due to the coupling with the ${ }^{14} \mathrm{~N}$ nuclear spin $(I=1)$, which has a natural abundance of $99.634 \%$. In addition, every carbon site in the crystal can be occupied by a ${ }^{13} \mathrm{C}$ nuclear spin $(I=1 / 2)$, which has a natural abundance of $1.1 \%$. Accordingly, many centers show unique spectra.

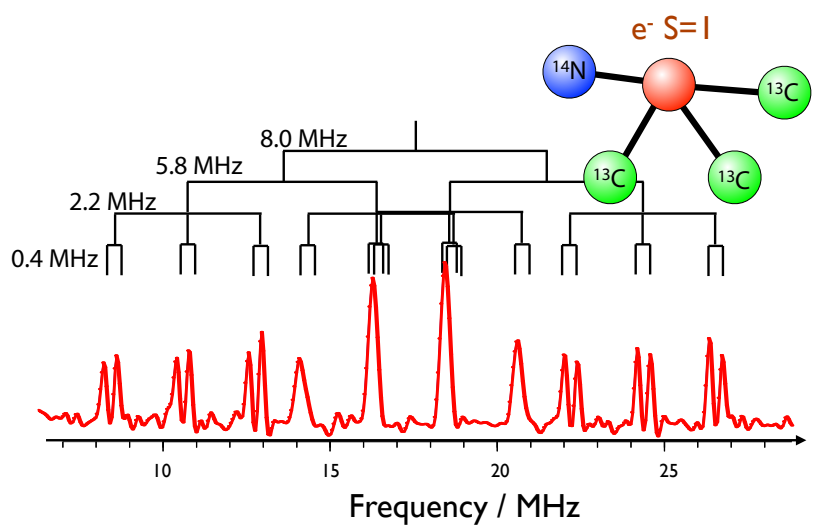

FIG. 11 Spectrum of a single electron spin coupled to three ${ }^{13} \mathrm{C}$ and one ${ }^{14} \mathrm{~N}$ nuclear spins.

Figure 11 shows, as an example, the spectrum of a center that is coupled to a single ${ }^{14} \mathrm{~N}$ nuclear spin and three different ${ }^{13} \mathrm{C}$ nuclear spins. Since the ${ }^{13} \mathrm{C}$ spins occupy different locations, their hyperfine coupling constants differ.

\section{Relaxation}

The case shown in Figure 11 is not a typical example. Most centers do not show resolved hyperfine couplings to ${ }^{13} \mathrm{C}$, but instead a broadening of the ESR lines resulting from the large number of hyperfine interactions that are $\ll 1 \mathrm{MHz}$. The number of these interactions depends on the concentration of ${ }^{13} \mathrm{C}$ nuclear spins. This has been verified, e.g., by comparing data from crystals with different isotopic compositions (Balasubramanian et al., 2009; Mizuochi et al., 2009). As shown in figure 12, the transverse relaxation times $T_{2}$ and $T_{2}^{*}$ show strong variations with the concentration of ${ }^{13} \mathrm{C}$ nuclear spins. In the case of $T_{2}^{*}$ (inverse linewidth), two different regimes of the hyperfine interaction can be distinguished: at high concentrations, the average distance between the electron and nuclear spins is relatively small. In this regime, the dominant contribution to the hyperfine interaction is the Fermi-contact term. At low concentrations, the average distance to the nuclear spins is large. In this regime, the Fermi-contact terms is negligible and the hyperfine interaction is dominated by the dipolar interaction.

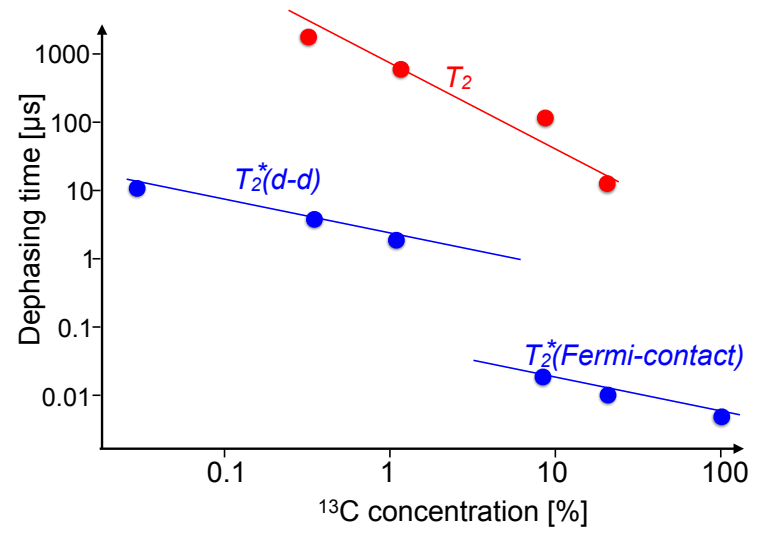

FIG. 12 Linewidth and relaxation time as a function of the ${ }^{13} \mathrm{C}$ nuclear spin concentration. $T_{2}^{*}(d-d)$ refers to the dephasing due to purely dipolar interactions. At higher concentration, the Fermi contact term dominates.

Apart from interactions with ${ }^{13} \mathrm{C}$ nuclear spins, couplings to paramagnetic centers also contribute to the relaxation rate. Which contribution dominates depends on different factors, such as the density of paramagnetic defects and the isotopic composition. In addition, the orientation of the magnetic field also affects the relaxation rate (Shin et al., 2013), particularly if the electron spin bath is the primary decoherence source.

Different mechanisms are responsible for longitudinal $\left(T_{1}\right)$-relaxation (Jarmola et al., 2012, 2015). At low densities and above room temperature, a two-phonon Raman process dominates, and below, an Orbach-type process, which involves the local vibrational modes of the NV center. At yet lower temperatures, the relaxation becomes temperature-independent, with time constants in the range of milliseconds to minutes, which depend on the details of the specific sample. In nitrogen-rich samples, dipolar couplings between electron spins drive cross-relaxation processes that reduce the measured $T_{1}$. If the density of defects is high enough, interactions between the centers can open additional relaxation channels (Jarmola et al., 2015).

\section{NUCLEAR MAGNETIC RESONANCE}

\section{A. Experimental Procedure}

The pulsed ESR experiment can be extended to the detection of NMR transitions of nuclear spins coupled to the electron spin.

Figure 13 shows the required sequence of pulses, together with the relevant energy level scheme for a system consisting of the electron spin and a single ${ }^{13} \mathrm{C}$ nuclear spin. The first laser pulse results in the population of the two $m_{S}=0, m_{I}= \pm 1 / 2$ spin states. As usual, $S$ 

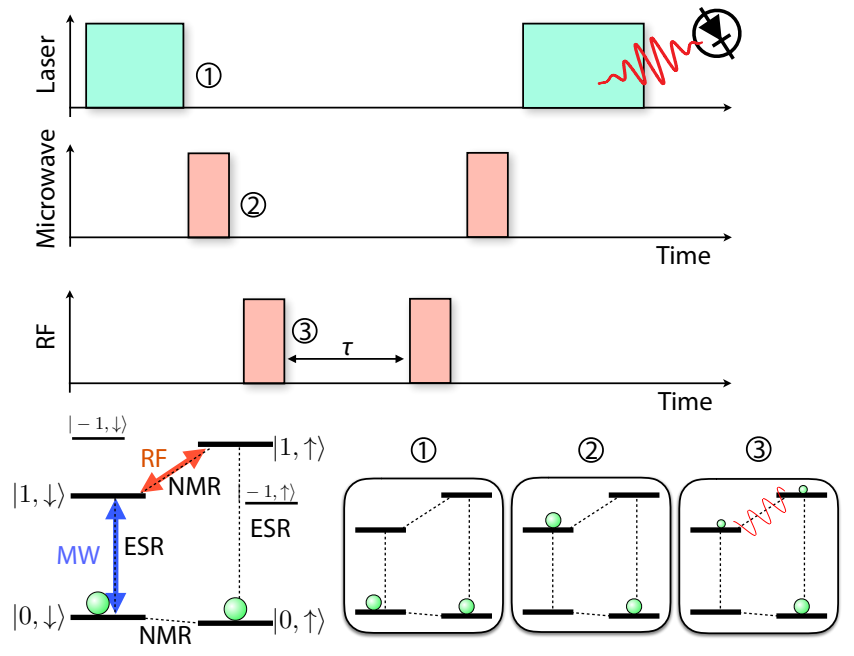

FIG. 13 Pulse sequence for optically detected NMR. The bottom part shows the relevant energy level scheme, together with a graphical representation of the 3 states that are generated during the sequence.

refers to the electron spin and $I$ to the nuclear spin. The corresponding system state is labeled (1) in the figure. A selective microwave pulse then exchanges the populations of the $m_{S}=0, m_{I}=1 / 2$ and $m_{S}=1, m_{I}=1 / 2$ states. In the resulting state (2), the two electron spin transitions and the two nuclear spin transitions are all equally polarized (apart from the sign). Applying a radio-frequency (RF)-pulse to one of the two nuclear spin transitions therefore can generate nuclear spin coherence. During the subsequent free-precession period of duration $\tau$, the nuclear spin coherence acquires a phase $\varphi=\omega_{N} \tau$ that encodes the nuclear spin transition frequency $\omega_{N}$. The final $\mathrm{RF}$ and microwave pulses convert this phase into modulation of the ground state population, which is then read out by the laser pulse.

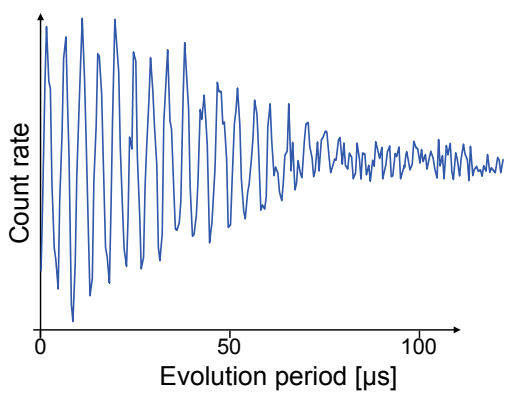

FIG. 14 Free-induction decay of a single ${ }^{13} \mathrm{C}$ nuclear spin located in the first shell of an NV center.

Figure 14 shows a typical example of a nuclear spin FID from a ${ }^{13} \mathrm{C}$ nuclear spin of the first coordination shell of an NV center. The dephasing time is relatively short for these nuclei, due to the strong interaction with the electron spin (see section IV.B).
The NV center also allows one to read out the actual state of a single nuclear spin (Neumann et al., 2010a) or even the state of several spins jointly (Dréau et al., 2013) and monitor them as they flip between different states.

\section{B. Nuclear Spin Interactions}

The possibility of exciting and detecting nuclear spin coherence hinges on the presence of hyperfine interactions between the electron and the nuclear spins. The interaction takes somewhat different forms for the two relevant nuclear spins, ${ }^{14} \mathrm{~N}$ and ${ }^{13} \mathrm{C}$.

\section{1. ${ }^{14} \mathrm{~N}$ Hyperfine Interaction}

The ${ }^{14} \mathrm{~N}$ nuclear spin is (almost) always present and interacts with the electron spin. Since the center has a 3 -fold rotational symmetry, the hyperfine interaction is axially symmetric, taking the form

$$
\mathcal{H}_{\mathrm{hfN}}=A_{||} I_{z} S_{z}+A_{\perp}\left(I_{+} S_{-}+I_{-} S_{+}\right) .
$$

The first term of this Hamiltonian commutes with the zero-field splitting $\mathcal{H}_{Z F}=D S_{z}^{2}$ of the electron spin and therefore has the dominant effect on the spectrum (see figure 15). The longitudinal component of the hyperfine coupling constant has the value $A_{\|} \approx-2.15 \mathrm{MHz}$. The transverse component $A_{\perp}$ does not commute with the zero-field Hamiltonian and therefore affects the energy levels only in second order.

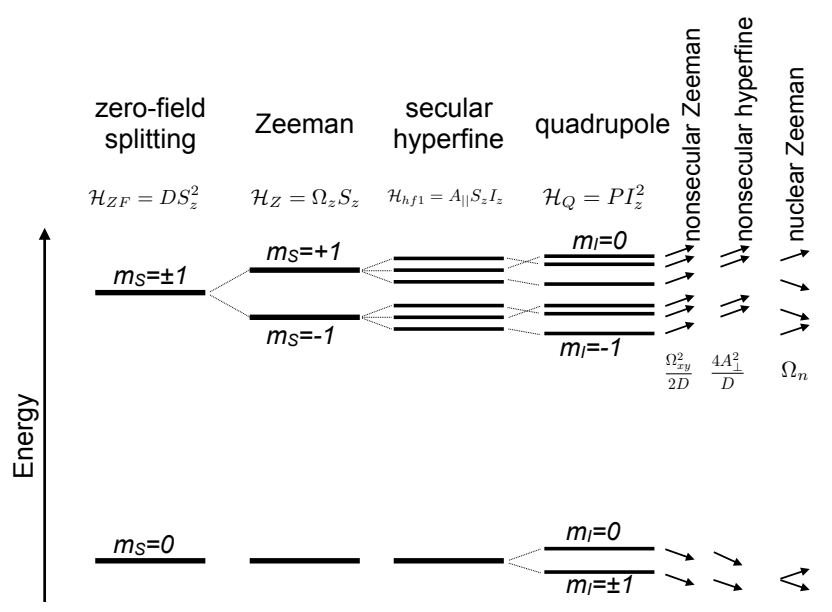

FIG. 15 Shifts and splittings of the energy levels of the electron spin $-{ }^{14} \mathrm{~N}$ nuclear spin system due to the different terms in the Hamiltonian. The arrows indicate the direction in which the corresponding Hamiltonian terms shift the energy levels. 


\section{2. ${ }^{14} \mathrm{~N}$ Quadrupole Interaction}

Since the ${ }^{14} \mathrm{~N}$ nucleus has a spin $I=1$, it is also subject to quadrupolar interaction. Since the center has a threefold symmetry axis, the quadrupole coupling can be written as

$$
\mathcal{H}_{Q}=P\left(S_{z}^{2}-\frac{2}{3} \mathbf{1}\right)
$$

As shown in figure 15, this splits the nuclear spin substates of the $m_{S}=0$ electron spin and changes the order of the energy levels in the $m_{S}= \pm 1$ multiplets. Further shifts are due to the nonsecular terms of the electron spin Zeeman interaction and the hyperfine interaction. The nuclear Zeeman term is some 4 orders of magnitude smaller than the electron Zeeman term and is usually only relevant when nuclear spin transitions are considered.

The NMR spectrum consists to first order of 3 resonance lines with frequencies $P$ and $P \pm A_{\|}$. With $P \approx 5$ $\mathrm{MHz}$ and $A_{\|} \approx 2.1 \mathrm{MHz}$, these transitions are close to 3 , 5 and $7 \mathrm{MHz}$.

\section{3. ${ }^{14} \mathrm{~N}$ nuclear Zeeman interaction}

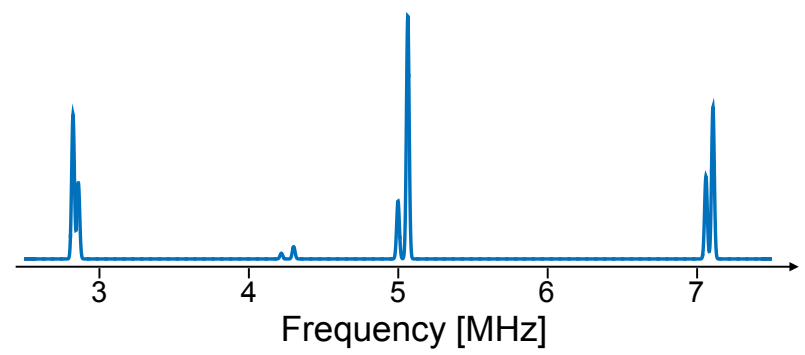

FIG. 16 Simulated NMR spectrum of the ${ }^{14} \mathrm{~N}$ nuclear spin in a $14.7 \mathrm{mT}$ field at an angle $\theta=64^{\circ}$ from the symmetry axis.

If the nuclear Zeeman interaction is also taken into account, the resonance lines are split, as shown in figure 16. Additional deviations from the simple picture include the fact that the transition dipole moments for the different transitions are not equal, which manifests itself by different amplitudes of the resonance lines. These variations are a consequence of the off-diagonal components of the hyperfine interaction, which mix the nuclear states in the different multiplets. In addition, two small transitions are visible close to $4.2 \mathrm{MHz}$. These are double quantum transitions $\left(m_{I}=-1 \leftrightarrow m_{I}=+1\right)$ for the $m_{S}= \pm 1$ multiplets. They become partially allowed since the nonsecular terms of the hyperfine interaction mix the eigenstates of $I_{z}$ and the nuclear and electronic spins are partially mixed. Accordingly, transitions within an electron spin multiplet also acquire a small contribution from the electronic spin (see also section IV.C). Since the electronic dipole moment is some 4 orders of magnitude larger than the nuclear moment, even a small admixture of electronic character can drastically change the transition strength. This additional contribution from the electronic spin has different signs for the different nuclear spin transitions. The total magnetic moment, which is the sum of the nuclear and electronic contributions, can therefore be smaller or larger than the magnetic moment of the bare nuclear spin. This explains the different transition amplitudes visible in figure 16. Conversely, the comparison between the different amplitudes can be used to determine the off-diagonal coupling constant (Chen et al., 2015a).

\section{4. ${ }^{13} \mathrm{C}$ Hyperfine}

We now consider a center, where one of the 3 carbons next to the vacancy is a ${ }^{13} \mathrm{C}$ and therefore carries a nuclear spin $1 / 2$. This site does not lie on the symmetry axis of the center. Accordingly, the symmetry of the hyperfine interaction is reduced to a single mirror plane that contains the nitrogen, the vacancy and the ${ }^{13} \mathrm{C}$ nucleus, as shown in fig. 17 .

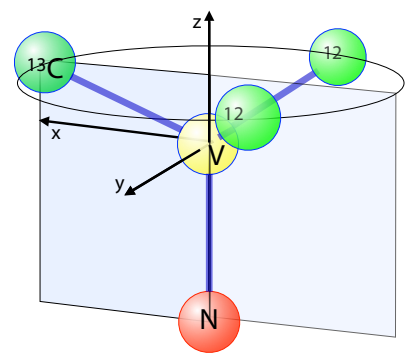

FIG. 17 Site symmetry of the NV center. The NV-axis lies along a $\mathrm{C}_{3}$-axis of the crystal, but if one of the carbon atoms is a ${ }^{13} \mathrm{C}$, the effective symmetry is reduced to $C_{s}$.

The hyperfine Hamiltonian for the ${ }^{13} \mathrm{C}$ nuclear spin can thus be written as

$\mathcal{H}_{\mathrm{hfC}}=A_{z z} S_{z} I_{z}+A_{x x} S_{x} I_{x}+A_{y y} S_{y} I_{y}+A_{x z}\left(S_{x} I_{z}+S_{z} I_{x}\right)$.

The other terms of the hyperfine tensor, which contain a single $y$-coordinate, vanish for symmetry reasons. The experimental values for the hyperfine coupling constants are $A_{x x}=189.3 \mathrm{MHz}, A_{y y}=128.4 \mathrm{MHz}$, $A_{z z}=128.9 \mathrm{MHz}$, and $A_{x z}=24.1 \mathrm{MHz}($ Rao and Suter, 2016). Again, the terms involving $S_{z}$ commute with the zero-field Hamiltonian $\mathcal{H}_{Z F}$ and therefore provide the dominant contribution to the energy of the spin states. For ${ }^{13} \mathrm{C}$ nuclear spins in the first shell, which have the strongest hyperfine interaction, the splitting is $\approx 127$ $\mathrm{MHz}$. For ${ }^{13} \mathrm{C}$ nuclear spins located at different positions of the diamond lattice, the hyperfine coupling decreases with distance. Many of the relevant coupling constants have been determined, either from quantum chemical 
calculations or from experimental data (Smeltzer et al., 2011).

Similar to the ${ }^{14} \mathrm{~N}$ case, the off-diagonal elements of the hyperfine interaction mix the different electronic and nuclear states. The eigenstates of the full Hamiltonian are therefore no longer eigenstates of the $S_{z}$ and $I_{z}$ operators. Accordingly, the selection rules for the ESR transitions are modified and the transition strengths of the different transitions depend on the amplitude and orientation of the magnetic field.

The non-secular component $A_{x z}$ of the hyperfine interaction is strong enough that the nuclear spin quantization axis is not the $z$-axis in small magnetic fields. It's direction depends on the quantum state of the electron spin: For $m_{S}= \pm 1$, it is oriented $\approx 11$ degrees from the $z$-axis in the $x z$-plane. In the $m_{S}=0$ state, the hyperfine interaction has a much weaker effect onto the eigenstates and the quantization axis is determined by the external magnetic field.

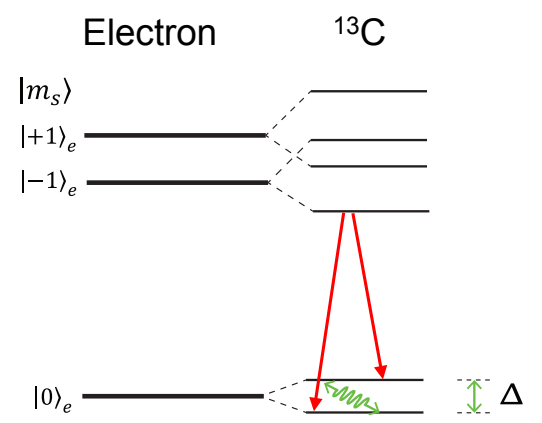

FIG. 18 Raman excitation of the ${ }^{13} \mathrm{C}$ nuclear transition associated with the $m_{S}=0$ state.

The different orientations of the quantization axes have also the consequence that the number of electron-spin transitions from $m_{S}=0$ to $m_{s}= \pm 1$ doubles: A direct excitation of the $|0,-1 / 2\rangle$ state into the $m_{S}=-1$ multiplet, e.g., generates a superposition of the $|-1,-1 / 2\rangle$ and $|-1,+1 / 2\rangle$ states. This means that the transition probability for both is nonzero. Similarly, the states $m_{S}=0$, $m_{I}= \pm 1 / 2$ both couple to all $m_{S}= \pm 1$ states, as shown in fig. 18 for one of those excited states. This fact can be used, e.g., to excite and detect the nuclear spin transition associated with the $m_{S}=0$ ground state using only microwave fields with frequencies close to the electron spin transitions labelled by the red arrows in Fig. 18. In a Raman-type process, these two fields can generate coherence in the $|0,-1 / 2\rangle \leftrightarrow|0,+1 / 2\rangle$ nuclear spin transition. The same type of Raman pulse can convert the coherence back into a population of the $m_{S}=0$ state, from where it can be read out in the usual way. It is thus possible to excite and detect nuclear spin transitions, whose transition frequencies are in the range of $0-10 \mathrm{MHz}$ without applying any radio frequency to the sample and without detecting radio-frequency signals being emitted by the sample.

\section{Enhanced Nuclear Magnetism}

Since the hyperfine interaction does not commute with the single-spin terms of the Hamiltonian, i.e. with the zero-field, quadrupolar and Zeeman interactions, the eigenstates of the system are not simple product states of the form $\left|m_{S}, m_{I}\right\rangle$, but they always contain an admixture of other basis states. Accordingly, transitions between them are neither pure electron-spin transition, where the electron spin changes by one unit, while the nuclear spin remains unchanged, nor pure nuclear-spin transitions, where the nuclear spin changes by one unit while the electron spin remains invariant.

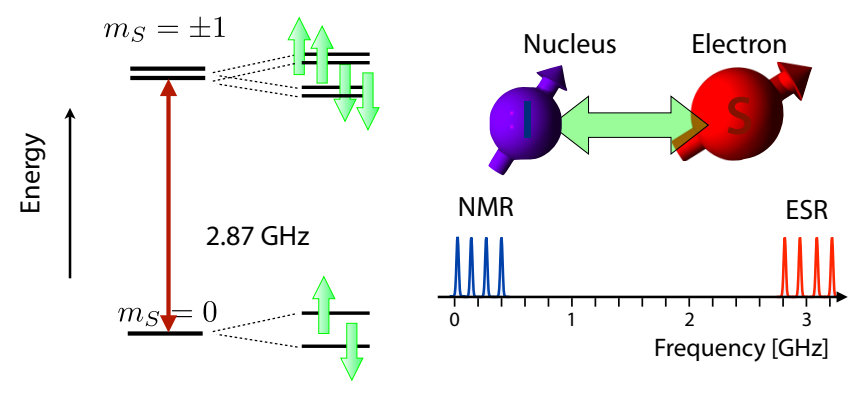

FIG. 19 Energy level scheme, spin states and transitions for a coupled electron-nuclear spin system.

For many applications, the labels $m_{S}$ and $m_{I}$ and 'NMR' vs. 'ESR' transitions remain good approximations. As shown in figure 19, in weak magnetic fields, the nominal NMR transitions are found at frequencies in the $\mathrm{MHz}$ range, while electron spin transitions are clustered around $2.9 \mathrm{GHz}$ for small magnetic fields. However, for strong hyperfine interactions, a nominal 'nuclear-spin' transition can include an admixture of up to $10 \%$ of electron spin character. Since the electron spin gyromagnetic ratio is some four orders of magnitude larger than that of both nuclear spins, this can have a very large effect.

The mixing of eigenstates is driven by the Hamiltonian terms $S_{x} I_{x}, S_{y} I_{y}$, and $S_{x} I_{z}$, which all have non-vanishing operator elements between the $m_{S}=0$ and the $m_{S}= \pm 1$ states. Since the off-diagonal elements are small compared to the zero-field splitting of $2.87 \mathrm{GHz}$, this can be analyzed by perturbation theory. Taking the off-diagonal elements into account, the $m_{S}=0$ state receives a small admixture of the $m_{S}= \pm 1$ states and the eigenstate of the full Hamiltonian can be written as

$$
|0\rangle^{\prime}=|0\rangle+\delta_{-}|-1\rangle+\delta_{+}|+1\rangle
$$

with $\left|\delta_{ \pm}\right| \ll 1$. These parameters depend on $m_{I}$ and therefore the transition between the substates also has electron spin character

While the admixture of electron spin character in these states remains small $(<10 \%)$, the change of the properties of the effective nuclear spin is highly significant. The simplest example is the effective Zeeman interaction of 


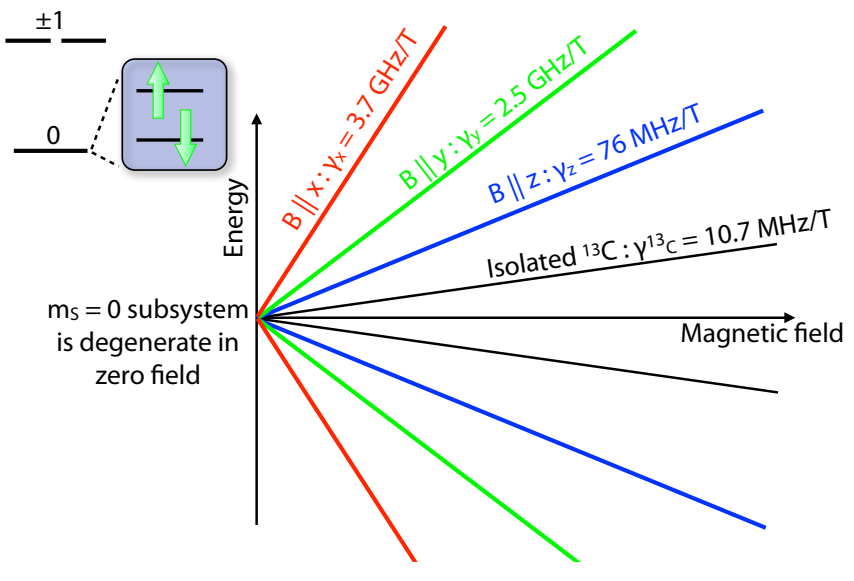

FIG. 20 Energy levels of the nuclear spin as a function of the magnetic field when it is coupled to the $\left|m_{S}=0\right\rangle$ state of the electron spin.

the nuclear spin when it is associated with the $\left|m_{S}=0\right\rangle$ state of the electron spin. In zero magnetic field, the nuclear $|\uparrow\rangle$ and $|\downarrow\rangle$ states are degenerate. An applied magnetic field splits the states linearly with the magnetic field, as usual for the Zeeman interaction. However, in this case, the splittings are much larger than for the bare nuclei, and the effective gyromagnetic ratio depends on the orientation of the magnetic field with respect to the symmetry axis of the center and to the symmetry plane of the $\mathrm{N}$-vacancy- ${ }^{13} \mathrm{C}$ system.

This result is closely analogous to the chemical shift: The hyperfine interaction, which does not commute with the electron-Zeeman interaction, modifies the nuclear Zeeman effect. The resulting splitting is therefore stronger than in the case of a bare nucleus. This effect can be described as an enhancement (or attenuation) of the effective local magnetic field, analogous to the chemical shift. However, compared to the common chemical shifts encountered in NMR of diamagnetic molecules, the effect is orders of magnitude larger. While the typical chemical shift range of ${ }^{13} \mathrm{C}$ in diamagnetic materials is $\approx 200 \mathrm{ppm}$, the isotropic part of the 'chemical shift' in the NV system is $-2 \cdot 10^{8} \mathrm{ppm}$ and the anisotropy is large (Rao and Suter, 2016): the principal values are $-351 \cdot 10^{6}$, $-241 \cdot 10^{6}$ or $-7.6 \cdot 10^{6} \mathrm{ppm}$ for magnetic fields aligned with the $x-, y-$ or $z$-axis.

The situation is different again, if the electron spin is in one of the $m_{S}= \pm 1$ states: in this case, the secular part of the hyperfine interaction splits the nuclear spin states by $\sqrt{A_{x z}^{2}+A_{z z}^{2}} \approx 131.1 \mathrm{MHz}^{1}$ (Rao and Suter, 2016). The hyperfine interaction, which is the dominant interaction, generates effective fields of $m_{S} \cdot 24.1 \mathrm{MHz}$ in the $x$-direction and $m_{S} \cdot 128.9 \mathrm{MHz}$ in the $z$-direction. The

1 The observed value is closer to $127 \mathrm{MHz}$, due to second-order effects quantization axis thus deviates from the $\pm z$-axis. In the absence of a magnetic field, the deviation is $\approx 10^{\circ}$. If a transverse magnetic field is applied that is strong compared to the hyperfine interaction, this field can become dominant and determine the quantization direction.

\section{QUANTUM INFORMATION}

The specific properties of the spins in diamond NVcenters (Doherty et al., 2013) make them interesting candidates for all tasks that rely on systems exhibiting specific quantum behavior (Nizovtsev et al., 2005). Applications range from the verification of basic quantum mechanical predictions like the Einstein-Podolsky-Rosen paradox (Hensen et al., 2015) to more practical goals in the field of quantum information (Stolze and Suter, 2008), where the NV system can be used, e.g., to test different types of quantum gate operations (Zhang et al., 2013).

Many interesting applications of quantum computing require many qubits (spins) that interact with one another. One approach for this is to use quantum registers consisting of several NV centers with spatial separations close enough so that their mutual dipole interaction is sufficiently strong for multi-qubit gate operations between them (Dolde et al., 2014; Neumann et al., 2010b; Yamamoto et al., 2013). While this approach has been tested with pairs of NV centers that were sufficiently close, it is unlikely that large arrays of NV centers can be generated with sufficient precision in the foreseeable future. NV centers cannot only be coupled directly, but also via electron spins that cannot be detected optically (Yao et al., 2012).

A second option is to use not only the electron spin, but to combine it with nuclear spins. In such hybrid quantum registers, the properties of the different types of qubits can show large differences. In particular, the gyromagnetic ratios differ by at least 3 orders of magnitude. Accordingly, the resonance frequencies, Rabi frequencies, and the decoherence times typically differ by several orders of magnitude. This can be used to advantage in some applications. As an example, since the decoherence time of nuclear spins can be orders of magnitude longer than that of electronic spins, it is usually advantageous to store quantum information in the nuclear spin degrees of freedom (Shim et al., 2013).

The different properties also imply a number of challenges: if operations must address electronic and nuclear spins at the same time, the evolution of the nuclear spin is typically some 3 orders of magnitude slower than that of the electronic spin. In many cases, the electronic spin is thus completely dephased before the operation on the nuclear spin is completed. This problem can be alleviated by applying refocussing pulses to the electron spin, using a procedure known as 'dynamical decoupling'(Suter and 
Álvarez, 2016; Viola et al., 1999) in the field of quantuminformation.

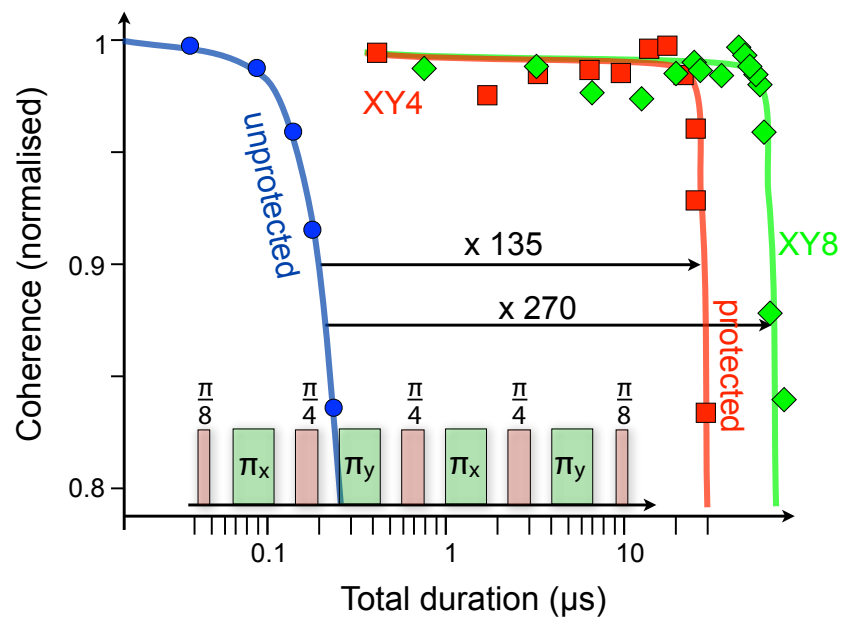

FIG. 21 Coherence remaining after a quantum gate operation that acts on a nuclear and an electronic spin as a function of the total duration of the gate. The data marked "unprotected" were obtained when only the pulses required by the gate operation were applied. For the protected gates, 4 and 8 additional refocusing pulses were used.

As shown in figure 21, the coherence of a hybrid quantum register consisting of an electron spin and a ${ }^{14} \mathrm{~N}$ nuclear spin decays over a timescale of a few $100 \mathrm{~ns}$ if no protective measures are taken. This timescale is given by the dephasing of the electron spin. It can be refocused, however, by applying a series of $\pi$-pulses to the electron spin, which refocus the effect of the environment (Shim et al., 2012; Zhang et al., 2014; Zhang and Suter, 2015). Such refocusing schemes can extend the dephasing time of the electron spin up to several ms at room temperature; at this point, it is limited by longitudinal $\left(T_{1^{-}}\right)$ relaxation (Shim et al., 2012).

Hybrid quantum registers based on an NV electron spin and external nuclear spins are the basis of a proposal for a diamond-based quantum simulator using arrays of nuclear spins on the surface of the diamond (Cai et al., 2013b). In addition, NV centers can be also interfaced with other types of qubits like photons (Togan et al., 2010), enabling the entanglement of distant qubits (Bernien et al., 2013). Ensembles of NV defects can be interfaced via magnetic coupling to superconducting qubits via superconducting resonators (Kubo et al., 2010; Sandner et al., 2012). These quantum interfaces will provide access to long spin coherence times of NV centers for other types of qubits enabling hybrid quantum registers combing the advantages of different quantum systems.

\section{SENSING}

The specific properties of the spins associated with the $\mathrm{NV}$ center have a number of interesting applications in the context of sensing - using single spins or ensembles of NV centers. Similar to the case of atomic magnetometers (Budker and Romalis, 2007), long living coherences of the electronic ground state of NV centers allow their use as nanoscale field sensors (Taylor et al., 2008).

The principle of operation of such a magnetometer can be understood as follows. The spin energy level structure associated with the ground state of NV centers can be probed using the ODMR techniques described in the previous sections of this review, and the measured frequency differences allow one to monitor changes in the magnetic field. Since power broadening reduces the sensitivity of cw measurements, pulsed experiments generally result in better performance. In the case of a (Ramseytype) FID measurement, the best sensitivity is achieved by measuring the phase accumulated by the spin over a period of the order of the decoherence time $T_{2}$ :

$$
\varphi=\gamma \int_{0}^{T_{2}} B(t) d t
$$

Longer acquisition times lead to loss of signal. Since a single measurement is not sufficient, it has to be repeated. For the total measurement time $\tau=n T_{2}$, with $n$ the number of repetitions, the standard quantum limit (in units of Tesla) for the sensitivity is

$$
\delta B=\frac{1}{\gamma} \frac{1}{\sqrt{T_{2} \tau}} .
$$

Since the photon collection efficiency is $<1$, the contrast and therefore the actual sensitivity is lower. Therefore techniques for improving the state detection efficiency in single measurements like auxiliary nuclear qubit assisted single shot readout or spin-to-charge conversion can be very important for sensing (Neumann et al., 2010a; Shields et al., 2015). For ensemble measurements involving $N$ independent NV centers, the sensitivity improves by a factor $\sqrt{N}$.

Several demonstrations of magnetometer protocols with single NV centers were published during the last decade. First experiments showed that single defects allow one to reach sensitivities on the order of $\approx \mu \mathrm{T} / \sqrt{\mathrm{Hz}}$ (Balasubramanian et al., 2008; Maze et al., 2008). Reducing the linewidth by use of isotopically enriched ${ }^{12} \mathrm{C}$ diamond improves the sensitivity up to a few $\mathrm{nT} / \sqrt{\mathrm{Hz}}$ (Balasubramanian et al., 2009).

NV centers can also sense alternating (ac) magnetic fields with high spectral resolution by employing dynamical decoupling (Álvarez and Suter, 2011; ?). Spin-echo sequences allow one to refocus the effect of external fields except for a particular frequency component. By careful design of the sensitivity function, it is possible to reach 
a spectral resolution of $\delta \nu=1 / T_{2}$. Dynamical decoupling sequences also can improve the sensitivity of diamond NV magnetometers by increasing the coherence time (de Lange et al., 2010). Although most demonstrations so far were obtained by application of pulsed dynamical decoupling techniques, it is also possible to use continuous driving techniques (Cai et al., 2013a, 2012).

A particularly interesting application of magnetometry is the detection of nuclear magnetic resonance on nanometer scales, including the detection of individual nuclear spins (Kolkowitz et al., 2012; Taminiau et al., 2012; Zhao et al., 2012) or small ensembles of external nuclear spins (Boss et al., 2016; Mamin et al., 2013; Staudacher et al., 2013). Single NV-centers currently have sufficient sensitivity for detecting single external nuclear spins and resolve their position within a few angstroms (Müller et al., 2014; Sushkov et al., 2014). The ability to bring the sensor close to biomolecules by implantation of single NV centers and attachment of proteins to the surface of diamond enabled the first proof of principle demonstration of proteins labelled by paramagnetic markers and label-free detection of the signal from a single protein (Lovchinsky et al., 2016).

Single-molecule NMR and MRI experiments open the way towards unravelling dynamics and structure of single biomolecules. However, for that purpose, NV magnetometers must reach spectral resolutions comparable to that of conventional solution state NMR. New techniques were proposed for this purpose and realized recently including correlation spectroscopy techniques (Kong et al., 2015; Laraoui et al., 2013) and approaches based on quantum memories (Zaiser et al., 2016). Quantum error correction protocols originally developed for protection of spin qubits in quantum processors were proposed (Arrad et al., 2014; Kessler et al., 2014) and shown to be able to improve both spectral resolution and sensitivity of diamond sensors by protecting them against high frequency noise including the case of high frequency noise where dynamical decoupling reaches its limits (Unden et al., 2016).

The ability to sense nuclear spins by NV centers also enables the transfer of polarization from optically polarized spins of NV centers to nuclear spins (King et al., 2015; London et al., 2013). Techniques for dynamic nuclear spin polarization (DNP) are very promising for the enhancement of sensitivity of conventional MRI imaging. Several approaches for NV centers enabled DNP were proposed including the use of diamond nanoparticles doped with ${ }^{13} \mathrm{C}$ nuclear spins as imaging agent (Chen et al., 2015b; King et al., 2015; Scheuer et al., 2016) or polarization transfer to external spins of liquids (Abrams et al., 2014; Chen et al., 2016).

The sensitivity of diamond magnetometers can also be improved by increasing the number of NV centers in the sensing volume. The sensitivity increases with the square root of the number of centers. Bulk NV mag- netometers were shown to be able to reach noise levels of $<1 \mathrm{pT} / \sqrt{\mathrm{Hz}}$ (Wolf et al., 2015). The use of infrared transitions between the singlet levels for magnetometry allows one to improve the sensitivity owing to higher contrast of the signal compared to conventional ODMR employing transitions between triplet levels for detection. The weakness of the IR transition can be compensated by a multi-pass technique enabled by optical resonators (Jensen et al., 2014). Ensemble magnetometry can be combined with wide field imaging (Steiner et al., 2010), enabling high efficiency parallel data acquisition including NMR detection with micrometer scale resolution (DeVience et al., 2015).

Single molecule NMR and ESR are not the only applications of NV centers for bio-sensing. The good biocompatibility of diamond surfaces opens the way for other applications of diamond sensors for life sciences including neuro-sensing (Barry et al., 2016; Hall et al., 2012), nanoscale measurement of electrochemical potential (Karaveli et al., 2016) and imaging bio-magnetic structures in living cells (Glenn et al., 2015; Le Sage et al., 2013). The ability to incorporate NV centers in nanodiamonds and the functionalization of diamond nanocrystals are key elements for the future development of diamond biosensors (see (Wu et al., 2016) for a recent review on bio-applications of nanodiamonds). Not only magnetic fields, but also other parameters like electric fields or temperature can influence the spin levels in $\mathrm{NV}$ centers, thus providing access to nano-thermometry and nano-electrometry measurements (Kucsko et al., 2013; Neumann et al., 2013). Hybrid sensors transferring electric fields or forces into magnetic fields and observing them with an NV center allows improved sensing protocols (Cai et al., 2014). In addition to sensing temperature, pressure, electrical and magnetic fields, the spins of the NV center can also be used to measure rotations and thereby form the basis of compact and sensitive gyroscopes (Ajoy and Cappellaro, 2012; Ledbetter et al., 2012; Maclaurin et al., 2012).

\section{CONCLUSIONS AND OUTLOOK}

The fascinating properties of the NV center have been studied in a wide range of contexts and not only allowed unique explorations in basic quantum mechanics, but also opened new options for quantum information processing, quantum communication and sensing. Given the rapid growth of this field, additional applications will certainly appear in the future. Of course, the NV-center of diamond is not the only defect in a solid, and many similar centers, in diamond as well as in other materials, are currently being explored and may well lead to other exciting opportunities. 


\section{ACKNOWLEDGMENTS}

Our work on this topic would not have been possible without a number of highly skilled and motivated group members, including J. H. Shim, I. Niemeyer, J. Zhang, K. R. K. Rao and T. Chakraborty. The research was supported by funding from the DFG through grants $\mathrm{Su}$ 192/27-1, 192/19-2, Su 192/31-1, SFB TR 21 and FOR 1493, by the DAAD, the Heinrich Hertz Foundation, by the MERCUR foundation through grant Pr-2013-0003, the ERC, VW Stiftung, and the BMBF.

\section{REFERENCES}

Abrams, D., M. E. Trusheim, D. R. Englund, M. D. Shattuck, and C. A. Meriles (2014), Nano Letters 14, 2471.

Acosta, V. M., A. Jarmola, E. Bauch, and D. Budker (2010), Phys. Rev. B 82, 201202.

Ajoy, A., and P. Cappellaro (2012), Phys. Rev. A 86, 062104.

Álvarez, G. A., and D. Suter (2011), Phys. Rev. Lett. 107, 230501.

Antonov, D., T. Häußermann, A. Aird, J. Roth, H.-R. Trebin, C. Müller, L. McGuinness, F. Jelezko, T. Yamamoto, J. Isoya, S. Pezzagna, J. Meijer, and J. Wrachtrup (2014), Applied Physics Letters 104, 012105.

Arrad, G., Y. Vinkler, D. Aharonov, and A. Retzker (2014), Phys. Rev. Lett. 112, 150801.

Balasubramanian, G., I. Y. Chan, R. Kolesov, M. Al-Hmoud, J. Tisler, C. Shin, C. Kim, A. Wojcik, P. R. Hemmer, A. Krueger, T. Hanke, A. Leitenstorfer, R. Bratschitsch, F. Jelezko, and J. Wrachtrup (2008), Nature 455, 648.

Balasubramanian, G., P. Neumann, D. Twitchen, M. Markham, R. Kolesov, N. Mizuochi, J. Isoya, J. Achard, J. Beck, J. Tissler, V. Jacques, P. R. Hemmer, F. Jelezko, and J. Wrachtrup (2009), Nature Materials 8, 383.

Barry, J. F., M. J. Turner, J. M. Schloss, D. R. Glenn, Y. Song, M. D. Lukin, H. Park, and R. L. Walsworth (2016), arXiv 1602.01056.

Batalov, A., C. Zierl, T. Gaebel, P. Neumann, I.-Y. Chan, G. Balasubramanian, P. R. Hemmer, F. Jelezko, and J. Wrachtrup (2008), Phys. Rev. Lett. 100, 077401.

Bernien, H., B. Hensen, W. Pfaff, G. Koolstra, M. S. Blok, L. Robledo, T. H. Taminiau, M. Markham, D. J. Twitchen, L. Childress, and R. Hanson (2013), Nature 497, 86.

Boss, J. M., K. Chang, J. Armijo, K. Cujia, T. Rosskopf, J. R. Maze, and C. L. Degen (2016), Phys. Rev. Lett. 116, 197601.

Budker, D., and M. Romalis (2007), Nat Phys 3, 227.

Bundy, F., H. Hall, H. Strong, and R. Wentorf (1955), Nature 176, 51 .

Cai, J., F. Jelezko, and M. B. Plenio (2014), Nature Communications 5, $4065 \mathrm{EP}$.

Cai, J., F. Jelezko, M. B. Plenio, and A. Retzker (2013a), New Journal of Physics 15, 013020.

Cai, J., A. Retzker, F. Jelezko, and M. B. Plenio (2013b), Nat Phys 9, 168.

Cai, J.-M., B. Naydenov, R. Pfeiffer, L. P. McGuinness, K. D. Jahnke, F. Jelezko, M. B. Plenio, and A. Retzker (2012), New Journal of Physics 14, 113023.
Chen, M., M. Hirose, and P. Cappellaro (2015a), Phys. Rev. B 92, 020101.

Chen, Q., I. Schwarz, F. Jelezko, A. Retzker, and M. B. Plenio (2015b), Phys. Rev. B 92, 184420.

Chen, Q., I. Schwarz, F. Jelezko, A. Retzker, and M. B. Plenio (2016), Phys. Rev. B 93, 060408.

DeVience, S. J., L. M. Pham, I. Lovchinsky, A. O. Sushkov, N. Bar-Gill, C. Belthangady, F. Casola, M. Corbett, H. Zhang, M. Lukin, H. Park, A. Yacoby, and R. L. Walsworth (2015), Nat Nano 10, 129.

Doherty, M. W., N. B. Manson, P. Delaney, F. Jelezko, J. Wrachtrup, and L. C. L. Hollenberg (2013), Physics Reports 528, 1.

Doi, Y., T. Fukui, H. Kato, T. Makino, S. Yamasaki, T. Tashima, H. Morishita, S. Miwa, F. Jelezko, Y. Suzuki, and N. Mizuochi (2016), Phys. Rev. B 93, 081203.

Dolde, F., V. Bergholm, Y. Wang, I. Jakobi, B. Naydenov, S. Pezzagna, J. Meijer, F. Jelezko, P. Neumann, T. SchulteHerbrüggen, J. Biamonte, and J. Wrachtrup (2014), Nature Communications 5, 3371.

Dréau, A., P. Spinicelli, J. R. Maze, J.-F. Roch, and V. Jacques (2013), Phys. Rev. Lett. 110, 060502.

Dumeige, Y., M. Chipaux, V. Jacques, F. Treussart, J.F. Roch, T. Debuisschert, V. M. Acosta, A. Jarmola, K. Jensen, P. Kehayias, and D. Budker (2013), Phys. Rev. B 87, 155202

Felton, S., A. M. Edmonds, M. E. Newton, P. M. Martineau, D. Fisher, and D. J. Twitchen (2008), Phys. Rev. B 77, 081201.

Glenn, D. R., K. Lee, H. Park, R. Weissleder, A. Yacoby, M. D. Lukin, H. Lee, R. L. Walsworth, and C. B. Connolly (2015), Nat Meth 12, 736.

Grangier, P., J. A. Levenson, and J.-P. Poizat (1998), Nature 396, 537 .

Gruber, A., A. Draebenstedt, C. Tietz, L. Fleury, J. Wrachtrup, and C. v. Borczyskowski (1997), Science 276, 2012.

Hall, L. T., G. C. G. Beart, E. A. Thomas, D. A. Simpson, L. P. McGuinness, J. H. Cole, J. H. Manton, R. E. Scholten, F. Jelezko, J. Wrachtrup, S. Petrou, and L. C. L. Hollenberg (2012), Sci. Rep. 2.

Harrison, J., M. Sellars, and N. Manson (2004), J. Luminesc. 107, 245.

Häußler, A. J., P. Heller, L. P. McGuinness, B. Naydenov, and F. Jelezko (2014), Opt. Express 22, 29986.

Hensen, B., H. Bernien, A. E. Dreau, A. Reiserer, N. Kalb, M. S. Blok, J. Ruitenberg, R. F. L. Vermeulen, R. N. Schouten, C. Abellan, W. Amaya, V. Pruneri, M. W. Mitchell, M. Markham, D. J. Twitchen, D. Elkouss, S. Wehner, T. H. Taminiau, and R. Hanson (2015), Nature 526, 682

Jarmola, A., V. M. Acosta, K. Jensen, S. Chemerisov, and D. Budker (2012), Phys. Rev. Lett. 108, 197601.

Jarmola, A., A. Berzins, J. Smits, K. Smits, J. Prikulis, F. Gahbauer, R. Ferber, D. Erts, M. Auzinsh, and D. Budker (2015), Applied Physics Letters 107, 242403, http://dx.doi.org/10.1063/1.4937489.

Jelezko, F., and J. Wrachtrup (2004), J. Phys.: Condens. Matter 16, R1089.

Jensen, K., N. Leefer, A. Jarmola, Y. Dumeige, V. M. Acosta, P. Kehayias, B. Patton, and D. Budker (2014), Phys. Rev. Lett. 112, 160802.

Karaveli, S., O. Gaathon, A. Wolcott, R. Sakakibara, O. A. Shemesh, D. S. Peterka, E. S. Boyden, J. S. Owen, 
R. Yuste, and D. Englund (2016), Proceedings of the National Academy of Sciences 113, 3938.

Kessler, E. M., I. Lovchinsky, A. O. Sushkov, and M. D. Lukin (2014), Phys. Rev. Lett. 112, 150802.

King, J. P., K. Jeong, C. C. Vassiliou, C. S. Shin, R. H. Page, C. E. Avalos, H.-J. Wang, and A. Pines (2015), Nature Communications 6, 8965 EP .

Köhler, J., J. Disselhorst, M. Donckers, E. Groenen, J. Schmidt, and W. Moerner (1993), Nature 363, 242.

Kolkowitz, S., Q. P. Unterreithmeier, S. D. Bennett, and M. D. Lukin (2012), Phys. Rev. Lett. 109, 137601.

Kong, X., A. Stark, J. Du, L. P. McGuinness, and F. Jelezko (2015), Phys. Rev. Applied 4, 024004.

Kubo, Y., F. R. Ong, P. Bertet, D. Vion, V. Jacques, D. Zheng, A. Dréau, J.-F. Roch, A. Auffeves, F. Jelezko, J. Wrachtrup, M. F. Barthe, P. Bergonzo, and D. Esteve (2010), Phys. Rev. Lett. 105, 140502.

Kucsko, G., P. C. Maurer, N. Y. Yao, M. Kubo, H. J. Noh, P. K. Lo, H. Park, and M. D. Lukin (2013), Nature 500, 54.

de Lange, G., Z. H. Wang, D. Riste, V. V. Dobrovitski, and R. Hanson (2010), Science 330, 60.

Laraoui, A., F. Dolde, C. Burk, F. Reinhard, J. Wrachtrup, and C. A. Meriles (2013), Nature Communications 4, 1651 EP.

Le Sage, D., K. Arai, D. R. Glenn, S. J. DeVience, L. M. Pham, L. Rahn-Lee, M. D. Lukin, A. Yacoby, A. Komeili, and R. L. Walsworth (2013), Nature 496, 486.

Ledbetter, M. P., K. Jensen, R. Fischer, A. Jarmola, and D. Budker (2012), Phys. Rev. A 86, 052116.

Lehtinen, O., B. Naydenov, P. Börner, K. Melentjevic, C. Müller, L. P. McGuinness, S. Pezzagna, J. Meijer, U. Kaiser, and F. Jelezko (2016), Phys. Rev. B 93, 035202.

London, P., J. Scheuer, J.-M. Cai, I. Schwarz, A. Retzker, M. B. Plenio, M. Katagiri, T. Teraji, S. Koizumi, J. Isoya, R. Fischer, L. P. McGuinness, B. Naydenov, and F. Jelezko (2013), Phys. Rev. Lett. 111, 067601.

Lovchinsky, I., A. O. Sushkov, E. Urbach, N. P. de Leon, S. Choi, K. De Greve, R. Evans, R. Gertner, E. Bersin, C. Müller, L. McGuinness, F. Jelezko, R. L. Walsworth, H. Park, and M. D. Lukin (2016), Science 351, 836.

Maclaurin, D., M. W. Doherty, L. C. L. Hollenberg, and A. M. Martin (2012), Phys. Rev. Lett. 108, 240403.

Mamin, H. J., M. Kim, M. H. Sherwood, C. T. Rettner, K. Ohno, D. D. Awschalom, and D. Rugar (2013), Science 339, 557.

Martin, J., R. Wannemacher, J. Teichert, L. Bischoff, and B. Köhler (1999), Applied Physics Letters 75, 3096.

Matsumoto, S., Y. Sato, M. Tsutsumi, and N. Setaka (1982), J. Materials Science 17, 3106.

Maze, J. R., P. L. Stanwix, J. S. Hodges, S. Hong, J. M. Taylor, P. Cappellaro, L. Jiang, M. V. G. Dutt, E. Togan, A. S. Zibrov, A. Yacoby, R. L. Walsworth, and M. D. Lukin (2008), Nature 455, 644.

Meijer, J., B. Burchard, M. Domhan, C. Wittmann, T. Gaebel, I. Popa, F. Jelezko, and J. Wrachtrup (2005), Applied Physics Letters 87, 261909.

Mizuochi, N., P. Neumann, F. Rempp, J. Beck, V. Jacques, P. Siyushev, K. Nakamura, D. J. Twitchen, H. Watanabe, S. Yamasaki, F. Jelezko, and J. Wrachtrup (2009), Phys. Rev. B 80, 041201.

Moerner, W., and L. Kador (1989), Phys. Rev. Lett. 62, 2535 .
Müller, C., X. Kong, J. M. Cai, K. Melentijevic, A. Stacey, M. Markham, D. Twitchen, J. Isoya, S. Pezzagna, J. Meijer, J. F. Du, M. B. Plenio, B. Naydenov, L. P. McGuinness, and F. Jelezko (2014), Nat Commun 5.

Neuhauser, W., M. Hohenstatt, P. E. Toschek, and H. Dehmelt (1980), Phys. Rev. A 22, 1137.

Neumann, P., J. Beck, M. Steiner, F. Rempp, H. Fedder, P. R. Hemmer, J. Wrachtrup, and F. Jelezko (2010a), Science 329,542 .

Neumann, P., I. Jakobi, F. Dolde, C. Burk, R. Reuter, G. Waldherr, J. Honert, T. Wolf, A. Brunner, J. H. Shim, D. Suter, H. Sumiya, J. Isoya, and J. Wrachtrup (2013), Nano Letters 13, 2738.

Neumann, P., R. Kolesov, V. Jacques, J. Beck, J. Tisler, A. Batalov, L. Rogers, N. B. Manson, G. Balasubramanian, F. Jelezko, and J. Wrachtrup (2009), New Journal of Physics 11, 013017 (10pp).

Neumann, P., R. Kolesov, B. Naydenov, J. Beck, F. Rempp, M. Steiner, V. Jacques, G. Balasubramanian, M. L. Markham, D. J. Twitchen, S. Pezzagna, J. Meijer, J. Twamley, F. Jelezko, and J. Wrachtrup (2010b), Nat Phys 6, 249.

Niemeyer, I., J. H. Shim, J. Zhang, D. Suter, T. Taniguchi, T. Teraji, H. Abe, S. Onoda, T. Yamamoto, T. Ohshima, J. Isoya, and F. Jelezko (2013), New J. Phys. 15, 033027.

Nizovtsev, A., S. Kilin, C. Tietz, F. Jelezko, and J. Wrachtrup (2001), Physica B 308-310, 608.

Nizovtsev, A. P., S. Y. Kilin, F. Jelezko, T. Gaebal, I. Popa, A. Gruber, and J. Wrachtrup (2005), Optics and Spectroscopy 99, 233.

Ohashi, K., T. Rosskopf, H. Watanabe, M. Loretz, Y. Tao, R. Hauert, S. Tomizawa, T. Ishikawa, J. Ishi-Hayase, S. Shikata, C. L. Degen, and K. M. Itoh (2013), Nano Letters 13, 4733.

Ohno, K., F. J. Heremans, L. C. Bassett, B. A. Myers, D. M. Toyli, A. C. B. Jayich, C. J. Palmstrom, and D. D. Awschalom (2012), Applied Physics Letters 101, 082413.

Fávaro de Oliveira, F., S. A. Momenzadeh, D. Antonov, J. Scharpf, C. Osterkamp, B. Naydenov, F. Jelezko, A. Denisenko, and J. Wrachtrup (2016), Nano Letters 16, 2228 .

van Oort, E., and M. Glasbeek (1991), Chemical Physics 152,365 .

van Oort, E., N. B. Manson, and M. Glasbeek (1988), J. Physics C: Solid State Physics 21, 4385.

Orrit, M., and J. Bernard (1990), Phys. Rev. Lett. 65, 2716.

Pezzagna, S., D. Rogalla, D. Wildanger, J. Meijer, and A. Zaitsev (2011), New Journal of Physics 13, 035024.

Pezzagna, S., D. Wildanger, P. Mazarov, A. D. Wieck, Y. Sarov, I. Rangelow, B. Naydenov, F. Jelezko, S. W. Hell, and J. Meijer (2010), Small 6, 2117.

Pham, L. M., S. J. DeVience, F. Casola, I. Lovchinsky, A. O. Sushkov, E. Bersin, J. Lee, E. Urbach, P. Cappellaro, H. Park, A. Yacoby, M. Lukin, and R. L. Walsworth (2016), Phys. Rev. B 93, 045425.

Rabeau, J. R., P. Reichart, G. Tamanyan, D. N. Jamieson, S. Prawer, F. Jelezko, T. Gaebel, I. Popa, M. Domhan, and J. Wrachtrup (2006), Appl. Phys. Lett. 88, 023113.

Ramsey, N. F. (1950), Phys. Rev. 78, 695.

Rao, K. R. K., and D. Suter (2016), Phys. Rev. B 94, 060101. Rittweger, E., K. Y. Han, S. E. Irvine, C. Eggeling, and S. W. Hell (2009), Nat Photon 3, 144.

Robledo, L., L. Childress, H. Bernien, B. Hensen, P. F. A. Alkemade, and R. Hanson (2011), Nature 477, 574. 
Sandner, K., H. Ritsch, R. Amsüss, C. Koller, T. Nöbauer, S. Putz, J. Schmiedmayer, and J. Majer (2012), Phys. Rev. A 85, 053806.

Santori, C., P. Tamarat, P. Neumann, J. Wrachtrup, D. Fattal, R. G. Beausoleil, J. Rabeau, P. Olivero, A. D. Greentree, S. Prawer, F. Jelezko, and P. Hemmer (2006), Phys. Rev. Lett. 97, 247401.

Scheuer, J., I. Schwartz, Q. Chen, D. Schulze-Sünninghausen, P. Carl, P. Höfer, A. Retzker, H. Sumiya, J. Isoya, B. Luy, M. B. Plenio, B. Naydenov, and F. Jelezko (2016), New Journal of Physics 18, 013040.

Schnitzler, W., N. M. Linke, R. Fickler, J. Meijer, F. SchmidtKaler, and K. Singer (2009), Phys. Rev. Lett. 102, 070501.

Schrödinger, E. (1952), Brit. J. Philos. Sci. 3, 233.

Shields, B. J., Q. P. Unterreithmeier, N. P. de Leon, H. Park, and M. D. Lukin (2015), Phys. Rev. Lett. 114, 136402.

Shim, J. H., I. Niemeyer, J. Zhang, and D. Suter (2012), Europhys. Lett. 99, 40004.

Shim, J. H., I. Niemeyer, J. Zhang, and D. Suter (2013), Phys. Rev. A 87, 012301.

Shin, C. S., C. E. Avalos, M. C. Butler, H.-J. Wang, S. J. Seltzer, R.-B. Liu, A. Pines, and V. S. Bajaj (2013), Phys. Rev. B 88, 161412.

Siyushev, P., H. Pinto, M. Vörös, A. Gali, F. Jelezko, and J. Wrachtrup (2013), Phys. Rev. Lett. 110, 167402.

Smeltzer, B., L. Childress, and A. Gali (2011), New Journal of Physics 13, 025021.

Spaeth, J.-M., J. Niklas, and R. Bartrm (1992), Structural analysis of point defects in solids (Springer).

Staudacher, T., F. Shi, S. Pezzagna, J. Meijer, J. Du, C. A. Meriles, F. Reinhard, and J. Wrachtrup (2013), Science 339,561 .

Steiner, M., P. Neumann, J. Beck, F. Jelezko, and J. Wrachtrup (2010), Phys. Rev. B 81, 035205.

Stepanov, V., F. H. Cho, C. Abeywardana, and S. Takahashi (2015), Applied Physics Letters 106, 063111.

Stolze, J., and D. Suter (2008), Quantum Computing: A Short Course from Theory to Experiment, 2nd ed. (Wiley$\mathrm{VCH}$, Berlin).

Sushkov, A. O., I. Lovchinsky, N. Chisholm, R. L. Walsworth, H. Park, and M. D. Lukin (2014), Phys. Rev. Lett. 113, 197601.

Suter, D., and G. A. Álvarez (2016), Rev. Mod. Phys. 88, 041001.

Taminiau, T. H., J. J. T. Wagenaar, T. van der Sar, F. Jelezko, V. V. Dobrovitski, and R. Hanson (2012), Phys. Rev. Lett. 109, 137602.
Taylor, J. M., P. Cappellaro, L. Childress, L. Jiang, D. Budker, P. R. Hemmer, A. Yacoby, R. Walsworth, and M. D. Lukin (2008), Nat Phys 4, 810.

Togan, E., Y. Chu, A. S. Trifonov, L. Jiang, J. Maze, L. Childress, M. V. G. Dutt, A. S. Sorensen, P. R. Hemmer, A. S. Zibrov, and M. D. Lukin (2010), Nature 466, 730.

Toyli, D. M., C. D. Weis, G. D. Fuchs, T. Schenkel, and D. D. Awschalom (2010), Nano Letters 10, 3168.

Unden, T., P. Balasubramanian, D. Louzon, Y. Vinkler, M. B. Plenio, M. Markham, D. Twitchen, A. Stacey, I. Lovchinsky, A. O. Sushkov, M. D. Lukin, A. Retzker, B. Naydenov, L. P. McGuinness, and F. Jelezko (2016), Phys. Rev. Lett. 116, 230502.

Viola, L., E. Knill, and S. Lloyd (1999), Phys. Rev. Lett. 82, 2417.

Wolf, T., P. Neumann, K. Nakamura, H. Sumiya, T. Ohshima, J. Isoya, and J. Wrachtrup (2015), Phys. Rev. X 5, 041001.

Wrachtrup, J., C. v. Borczyskowski, J. Bernard, M. Orrit, and R. Brown (1993), Nature 363, 244.

Wrachtrup, J., A. Gruber, L. Fleury, and C. v. Borczyskowski (1997), Chem. Phys. Lett. 267, 179.

Wu, Y., F. Jelezko, M. B. Plenio, and T. Weil (2016), Angewandte Chemie International Edition 55, 6586.

Yale, C. G., B. B. Buckley, D. J. Christle, G. Burkard, F. J. Heremans, L. C. Bassett, and D. D. Awschalom (2013), Proceedings of the National Academy of Sciences of the United States of America 110, 7595.

Yamamoto, T., C. Müller, L. P. McGuinness, T. Teraji, B. Naydenov, S. Onoda, T. Ohshima, J. Wrachtrup, F. Jelezko, and J. Isoya (2013), Phys. Rev. B 88, 201201.

Yao, N. Y., L. Jiang, A. V. Gorshkov, P. C. Maurer, G. Giedke, J. I. Cirac, and M. D. Lukin (2012), Nature Communications 3, 800 EP .

Zaiser, S., T. Rendler, I. Jakobi, T. Wolf, S.-Y. Lee, S. Wagner, V. Bergholm, T. Schulte-Herbrüggen, P. Neumann, and J. Wrachtrup (2016), Nature Communications 7, 12279 $\mathrm{EP}$.

Zaitsev, A. (2001), Optical properties of diamond (Springer). Zaitsev, A. M. (2000), Phys. Rev. B 61, 12909.

Zhang, J., J. H. Shim, I. Niemeyer, T. Taniguchi, T. Teraji, H. Abe, S. Onoda, T. Yamamoto, T. Ohshima, J. Isoya, and D. Suter (2013), Phys. Rev. Lett. 110, 240501.

Zhang, J., A. M. Souza, F. D. Brandao, and D. Suter (2014), Phys. Rev. Lett. 112, 050502.

Zhang, J., and D. Suter (2015), Phys. Rev. Lett. 115, 110502. Zhao, N., J. Honert, B. Schmid, M. Klas, J. Isoya, M. Markham, D. Twitchen, F. Jelezko, R.-B. Liu, H. Fedder, and J. Wrachtrup (2012), Nat Nano 7, 657. 


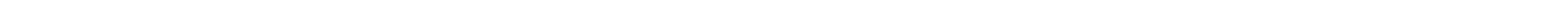

